\title{
La lengua de Caritón de Afrodisias: características morfológicas*
}

\author{
Manuel Sanz Morales \\ Universidad de Extremadura \\ msanz@unex.es
}

Recibido: $30-11-2014$

Aceptado: 16-12-2014

\section{RESUMEN}

Este trabajo es el primero que estudia de forma global las características morfológicas de la lengua de Caritón. Tras un análisis pormenorizado de cada uno de los rasgos pertinentes, se concluye que Caritón no usa una lengua aticista, ya que son predominantes los rasgos propios de la koiné helenística, unos coexistiendo con los correspondientes rasgos áticos, pero otros con carácter exclusivo. Sin embargo, existen también algunos rasgos exclusivos del ático, si bien este hecho no se da de manera sistemática. Ello lleva a una segunda conclusión, consistente en que la prosa de Caritón muestra características clasicistas, sin que pueda ser considerada aún prosa aticista.

Palabras clave: Caritón de Afrodisias, historia de la lengua griega, aticismo, koiné helenística, morfología griega.

\begin{abstract}
The present contribution is the first to study the morphological characteristics of Chariton of Aphrodisias's language as a whole. After a thorough analysis of each relevant feature, it can be concluded that Chariton does not use an atticist language, because the features of the Hellenistic koine are predominant: while some of them co-exist with the corresponding attic traits, others are exclusive for the koine. However, some exclusive attic features can be found as well, although not on a systematic basis. This allows us to draw a second conclusion: Chariton's prose shows classicist characteristics, but it cannot be considered atticist prose yet.
\end{abstract}

Keywords: Chariton of Aphrodisias, history of the Greek language, atticism, Hellenistic koine, Greek morphology.

0. Este artículo estudia las características morfológicas de la lengua de Caritón, un aspecto de su lengua que, a diferencia de otros, apenas ha sido tratado hasta ahora por los especialistas. ${ }^{1}$ La única obra que se ha ocupado de ello, el libro de Papanikolaou

* Agradezco a mi colega Helena Maquieira Rodríguez las valiosas sugerencias hechas a este trabajo.

${ }^{1}$ No me detendré en pormenores acerca de los trabajos de investigación sobre sintaxis y léxico, cuestión sobre la que remito a una breve panorámica ya publicada (Sanz Morales 2014: 291-292); en ese mismo artículo se estudian las características principales de la fonología (Sanz Morales 2014: passim). 
(1973), en realidad se limita a problemas muy específicos, ya que presta la mayor parte de su atención al léxico y, sobre todo, a la sintaxis.

En el presente trabajo analizaré de manera individual los principales rasgos morfológicos ${ }^{2}$ que pueden resultar pertinentes para definir y situar la lengua de Caritón en relación con las dos corrientes que, de una manera general, caracterizan el griego literario en los primeros siglos de nuestra era: la koiné propia de su época, por un lado, y el aticismo o movimiento cultista y arcaizante que postulaba un regreso a la lengua literaria de los autores del periodo clásico. A modo de primera conclusión, y a la luz de los resultados del estudio de los rasgos individuales, trataré de situar la lengua de Caritón (en el aspecto morfológico) en su contexto lingüístico histórico. Después, y como segunda conclusión, pondré ese primer resultado general en relación con los resultados obtenidos por investigaciones anteriores con respecto a la fonología, la sintaxis y el léxico.

Salvo indicación expresa, para citar el texto de Caritón seguiré la edición de Reardon (2004). ${ }^{3}$

\section{MORFOLOGÍA NOMINAL}

\subsection{Temas en $-O$}

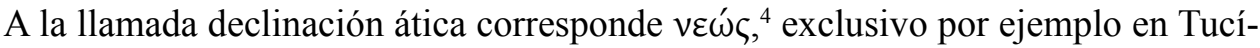
dides. vaó, forma dórica presente ya en la tragedia, se impone después en la koiné, siendo la normal en los papiros, así como exclusiva en el $N T$. En Caritón se dan ambas

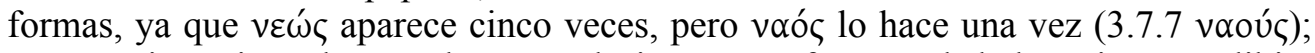
esta coexistencia se da ya en la época clásica en Jenofonte, en la helenística en Polibio,

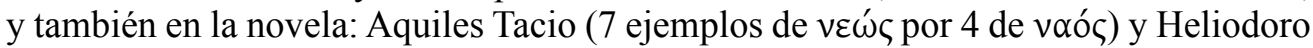
( 29 y 5 , más un caso de vnós en una cita). ${ }^{5}$

${ }^{2}$ La elección de rasgos se basa en estudios anteriores acerca de la lengua de otros autores griegos: Weissenberger (1994) [= 1895], Vela Tejada (1991), Caballero López (1997) y, especialmente (por su abundancia de datos y por estudiar una obra probablemente coetánea de la de Caritón), Vicente Sánchez (2006).

${ }^{3}$ Es también la edición utilizada por el $T L G$. Si es necesario, tendré presentes las demás ediciones aparecidas hasta la fecha: D’Orville (1783²), Hirschig (1856), Hercher (1859), Blake (1938), Molinié $\left(1989^{2}\right)$, Goold (1995), Borgogno (2005) y Meckelnborg-Schäfer (2006). El comienzo de este trabajo puede ser un buen lugar para hacer una precisión metodológica (repito lo ya dicho con respecto a la fonología, cf. Sanz Morales 2014: 292, n. 4). Lo que está en nuestra mano analizar no es el sistema morfológico real de Caritón, como hablante de la koiné de su época, sino el proporcionado por la lengua escrita con que él pretende reproducir (o no) la de los antiguos autores áticos. Estamos, pues, ante fenómenos estilísticos o de moda literaria, que como hechos lingüísticos pertenecen solo a la prosa literaria (Crespo 1979-80: 109 y 111-112). En relación con esto, es necesario asimismo tener presente la influencia de las posibles interferencias debidas a la transmisión textual (Crespo 1979-80: 110; Sanz Morales 2014: 292, n. 4).

${ }^{4}$ Es de rigor hacer notar que el estudio roza el campo del léxico con relación a esta y algunas otras palabras, por ser únicas o casi únicas dentro de su categoría morfológica.

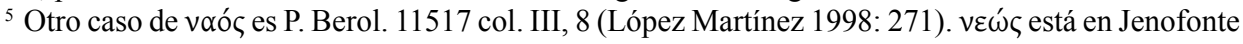
de Éfeso $(1 \times)$ y Longo $(6 \times)$, así como en un papiro, PSI 726 col. I, 6. 
Los sustantivos en - $\alpha \rho \chi 0 \varsigma$, que predominan en las inscripciones y autores de época clásica, van siendo sustituidos por las formas de origen jónico en - $\alpha \rho \chi \eta \varsigma$, que a su vez

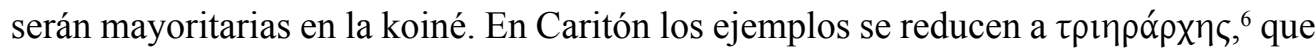
aparece 5 veces, solo en esta forma. ${ }^{7}$ Se trata, pues, de un rasgo de koiné; es digno de mención que Tucídides y Jenofonte, los principales autores áticos que influyen en

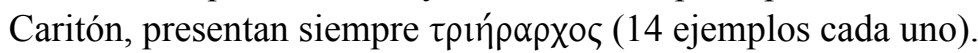

Con respecto a la flexión contracta, cuestión que ya ha sido estudiada anteriormente como manifestación del rasgo fonológico de la contracción, ${ }^{8}$ hay que decir que Caritón presenta tanto formas contractas como sin contraer, situación habitual en la

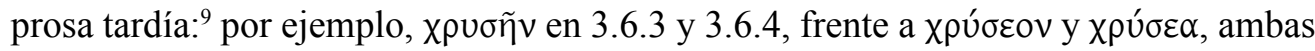
palabras en 6.4.2 (la primera repetida)..$^{10}$ Dado que los autores aticistas prefieren las formas contractas, de acuerdo con el uso ático, parece que Caritón no sigue en este punto la norma aticista.

\subsection{Temas en - $a$}

En autores áticos como Tucídides o Jenofonte aparece en ocasiones el locativo

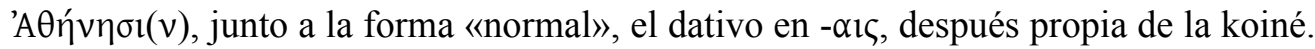

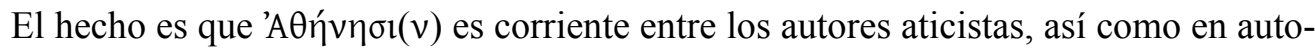
res u obras posiblemente coetáneos de Caritón, como Plutarco ( $L y c$. 24.3, etc.) o las Cartas de Temístocles (1.2, etc.). Sin embargo, el único ejemplo posible de Caritón

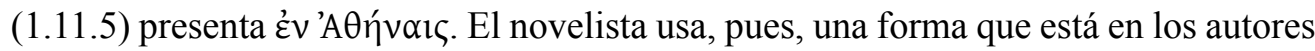
áticos, pero que es normal en la koiné, prefiriéndola a la forma aticista.

\subsection{Declinación atemática}

\subsubsection{Temas en dental}

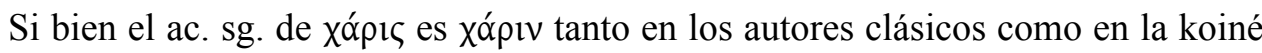

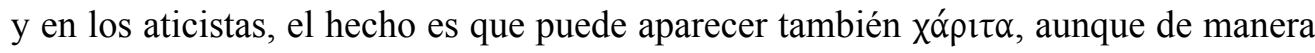

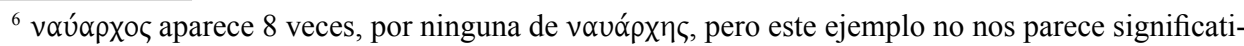

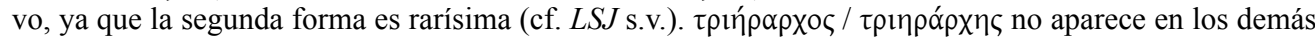
novelistas.

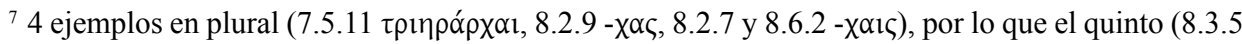

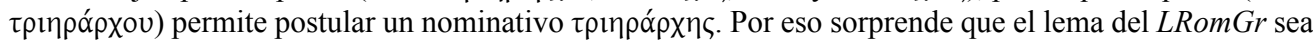

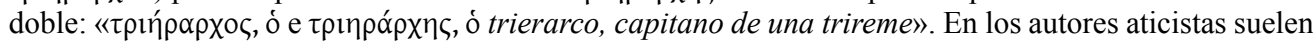

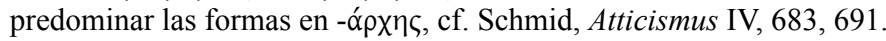

${ }^{8}$ Sanz Morales (2014: 296).

${ }^{9}$ Así sucede en los papiros de época imperial (Gignac 1981: 33 ss., 116 ss).

${ }^{10}$ Encontramos un panorama semejante en otras categorías morfológicas, por ejemplo $\pi \rho 0 u ́ \delta \omega \kappa \varepsilon$ $(3.2 .13,3.7 .4)$ frente a $\pi \rho \circ \varepsilon ́ \delta \omega \kappa \varepsilon(5.6 .3,6.7 .9)$, etc. 
muy minoritaria. ${ }^{11}$ No es el caso de Caritón, que presenta el esperable xópıv en 38 ocasiones frente a ningún ejemplo de $\chi \alpha{ }^{\rho} \rho \imath \alpha .{ }^{12}$

\subsubsection{Temas en $-u$}

Merece ser mencionado el gen. sg. ớ Esta forma, que no está en los papiros ptolemaicos ni en el $N T,{ }^{13}$ puede darse en los autores contemporáneos de Caritón y en los aticistas: respectivamente, hay ejemplos en Dionisio de Halicarnaso, Filón de Alejandría y Flavio Josefo, y en Luciano, Elio

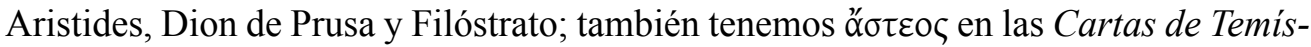
tocles $4.10 .{ }^{14}$ Mientras que esta es una forma épica y jónica, la forma ática es ä $\sigma \tau \varepsilon \omega \varsigma,{ }^{15}$ única que tenemos en escritores clásicos y modelos de Caritón, como son Tucídides $(3 \times)$ y Jenofonte $(16 \times)$, pero que no está en el novelista. ${ }^{16}$ No resulta fácil explicar la

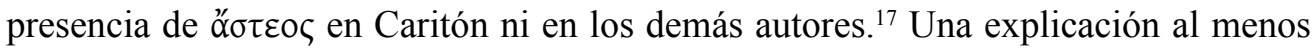
estimable es la que da Vicente Sánchez (2006: 113) con respecto al ya mencionado ejemplo de Themist. 4.10: dado que - $\varepsilon \omega \varsigma$, que ya estaba en los sustantivos, se extiende

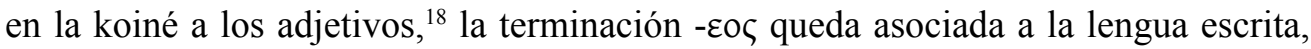
ya que es propia de la poesía, por lo que los autores buscan mediante ella un nivel más elevado para su lengua. El hecho de que la palabra sea una rareza en los papiros ptolemaicos y no se use en el $N T$, y por tanto haya quedado como vocablo puramente literario, podría haber influido en el fenómeno.

\subsubsection{Temas en diptongo}

El nom. pl. de $\beta \alpha \sigma \imath \lambda \varepsilon v ́ \varsigma$ aparece en Caritón 4 veces como $\beta \alpha \sigma \imath \lambda \varepsilon \tilde{\imath} \varsigma$, una forma normal en los autores áticos (exclusiva en Platón, 14×, y Jenofonte, $8 \times$ ), si bien estos autores pueden presentar la forma antigua $\beta \alpha \sigma \lambda \lambda \tilde{\eta} \varsigma$ (Tucídides, en la única aparición

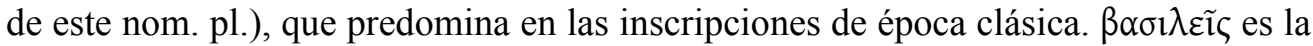
forma de la koiné.

${ }^{11}$ Un ejemplo ya en Jenofonte, Hel. 3.5.16, frente a 61 ejemplos de xópıv. En el NT reaparece: Act. 24.27 (v.l. xápıv) y Iudae 4; cf. Bl.-D.-R. §47.3 y 47, que la incluyen como ejemplo de la recuperación tardohelenística de formas en dental, junto a $\kappa \lambda \varepsilon \tilde{\imath} \delta \alpha$ y $\kappa \lambda \varepsilon \tilde{\imath} \delta \alpha \varsigma$. Cf. también Mayser I, 2, p. 31.

${ }^{12}$ Lo mismo sucede en los otros cuatro novelistas, con 36 ejemplos de xápıv en total.

${ }^{13}$ En los papiros aparece ó $\sigma \tau \varepsilon \omega \varsigma$ (Mayser I, 2, p. 25), mientras que el NT no documenta el término, como puede verse en Bauer-Aland.

${ }_{14}^{14}$ Para todo ello, cf. Vicente Sánchez (2006:113).

${ }^{15}$ La que aparece en las inscripciones: K.-B1. I, 441; Threatte (1996:217).

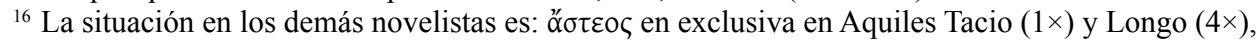

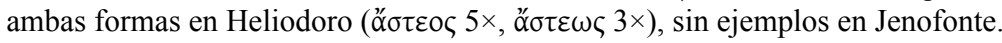

${ }^{17}$ Vela Tejada (1991: 127) explica su presencia en Eneas Táctico (siglo IV a.C.), como influencia lite-

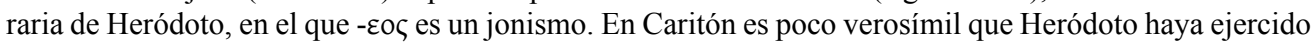
esa influencia por encima de Tucídides o Jenofonte, autores mucho más presentes en el novelista.

${ }^{18}$ Chantraine, Morph. $\$ 91$. 
La palabra vaṽ, que en época tardía se convierte en un término literario (los papiros y el $N T$ utilizan $\pi \lambda$ oĩov), ${ }^{19}$ aparece 27 veces en Caritón, en todos los casos salvo el gen. pl. Presenta la declinación propia del ático, excepto en las dos apariciones del

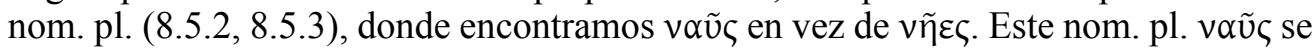
encuentra también en ático, con ejemplos en inscripciones, ${ }^{20} \mathrm{y}$ aparece tardíamente en autores aticistas como Eliano. ${ }^{21}$ Sin embargo, autores de léxicos aticistas como Frínico o Meris la condenan o contraponen al correcto $v \tilde{\eta} \varepsilon \varphi^{22}$ Sea cual sea el motivo del uso de ese nominativo, el hecho es que Caritón no se aparta aquí de la norma ática en la medida en que no usa formas que puedan atribuirse a la koiné, formas de raigambre jónica. Es destacable que el novelista no mezcle las formas áticas con las jónicas, a diferencia de lo que sucede en los autores mencionados o, ya antes, en la koiné literaria

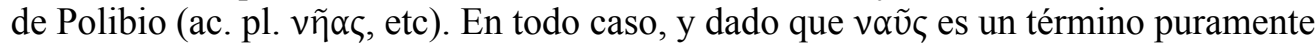
literario, como indicábamos antes, no debe extrañar la situación descrita.

\subsection{Dual}

Como es sabido, en la koiné el número dual desaparece de la flexión nominal y verbal. ${ }^{23}$ Después, reaparecerá con cierta frecuencia en los autores aticistas, quienes lo resucitarán artificialmente. ${ }^{24}$ Caritón no lo usa en la flexión nominal o pronominal, con

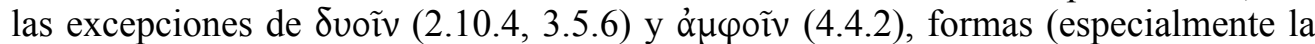
primera) cuyo valor como rasgos genuinos de dual es discutible. ${ }^{25}$ Es de interés comparar esta situación con la que se da en otros dos novelistas: a diferencia de Caritón, tanto Aquiles Tacio como Heliodoro presentan varios ejemplos de dual, casi siempre

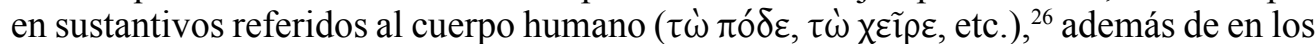
dos pronombres ya mencionados. ${ }^{27}$ Está claro que Caritón no incorpora un aticismo, que, además, está presente después en otros autores del mismo género literario.

\subsection{Comparativo y superlativo}

Una cuestión morfológica que merece atención en el apartado de los comparativos es la de los casos que pueden llevar, o bien sufijo sigmático, ${ }^{28}$ lo que da lugar a formas

${ }^{19}$ Mayser I, 2, p. 28, Gignac (1981: 82); un único ejemplo de vaṽ $\varsigma$ en el NT: Bl.-D.-R. $§ 47.4 d$ y $47_{8}$,

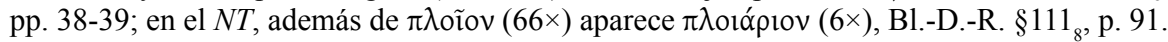

${ }^{20}$ Threatte (1996: 228).

${ }^{21}$ Ael., $N A$ 10.17.15, 13.28.5, 14.26.4, 15.5.16. Como Caritón, por lo demás sigue la norma ática. Vicente Sánchez (2006: 112) afirma que hay ejemplos en Polemón, pero no he podido encontrar ninguno.

${ }^{22}$ Threatte (1996: 228-229).

${ }^{23}$ Mayser II, 1, p. 34; B1.-D.-R. §2, 2 a y 65.

${ }^{24}$ Para las formas nominales y pronominales, cf. Schmid, Atticismus I, 87, 233; II, 35; III, 46-48; IV, 43-46.

${ }^{25}$ Con respecto a estas dos formas, véase el apdo. 1.6.4.

${ }^{26}$ Esto ocurre ya en Polibio, que solo atestigua formas en -oĩv (cf. Foucault 1972: 69).

${ }^{27}$ En Jenofonte de Éfeso y Longo, en cambio, la situación es similar a la de Caritón. Datos completos en Papanikolaou (1973: 93-94).

${ }^{28}$ Sobre este sufijo primario, uno de los arcaísmos presentes en el dialecto ático, cf. Chantraine, Morph., §112. 


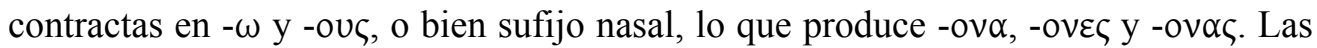
formas contractas son habituales en ático clásico, siendo mayoritarias en las inscripciones. ${ }^{29}$ Las dos posibilidades se dan también en los autores clásicos y, si bien en algunos de ellos predominan claramente las formas contractas, como es el caso de Tucídides ${ }^{30}$ el hecho es que ambas coexisten; y parece que coexistían también en el ático coloquial, ya que ambas aparecen en las comedias aristofánicas. ${ }^{31}$ Después, el sufijo nasal se va imponiendo, lo que ya ocurre en la koiné y es más claro en el NT.

Así que parece lógico que en los autores aticistas se dé el fenómeno contrario, y que por tanto predominen en ellos las formas contractas, consideradas genuinamente áticas; igual ocurre en una obra tardía y quizá contemporánea de Caritón como las Cartas de Temistocles. ${ }^{32}$

En Caritón, por el contrario, el panorama es diferente al de los aticistas, ya que las formas con el sufijo nasal son mayoría (19 ejemplos) frente a las contractas (4 ejemplos). ${ }^{33}$

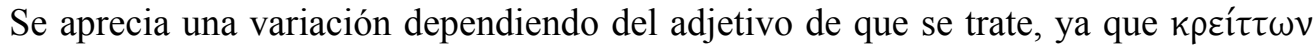
$(5 \times$ ac. sg., $1 \times$ nom. pl. $)$ y $\mu \varepsilon i ́ \zeta \omega \nu(2 \times$ ac. sg., $1 \times$ ac. pl. neutro, $1 \times$ ac. pl. masc. fem. $)$

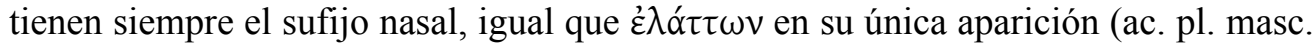
fem.), en tanto que $\beta \varepsilon \lambda \tau i ́ \omega v$ varía en solo dos ejemplos, presentando $\beta \varepsilon \lambda \tau i ́ \omega$ en ac. sg. (5.7.2) у $\beta \varepsilon \lambda \tau i ́ o v \alpha \varsigma$ en ac. pl. (6.9.8); la mayor variedad se da en $\pi \lambda \varepsilon i ́ \omega v: \pi \lambda \varepsilon i ́ \omega$ ac.

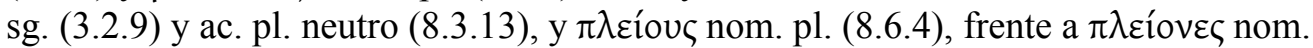

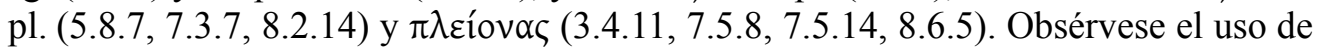
$\pi \lambda \varepsilon$ cíou y $\pi \lambda \varepsilon$ zíoveৎ para el mismo caso, nom. pl. masculino o femenino.

En conjunto, parece que Caritón sigue la tendencia dominante en la koiné y en general en el griego tardío, pero sin desterrar por completo las formas consideradas áticas, que en este caso son las contractas.

\subsection{Pronombres}

\subsubsection{Pronombres reflexivos}

Una primera cuestión digna de mención es la sustitución del plural de $3 .^{\mathrm{a}}$ persona $\sigma \varphi \tilde{\omega} v \alpha u \dot{\tau} \tilde{\omega} v$, etc. por las formas secundarias $\dot{\varepsilon} \alpha u \tau \tilde{\omega} v$, etc., en analogía con el sg. $\dot{\varepsilon} \alpha u \tau o \tilde{v}$. Ello sucede ya en las inscripciones, sobre todo después de ca. 400 a.C. ${ }^{34}$ y se da asimismo en autores de época clásica como los poetas trágicos, Tucídides, Platón, Isócrates, etc. Más adelante lo tenemos en $\operatorname{los}_{\text {papiros }}{ }^{35}$ e igualmente en los autores

${ }^{29}$ Threatte (1996: 311-312). En el siglo II a.C. las formas en -ov- son comunes, pero las contractas se siguen usando; este uso continúa, aunque atenuado, en época romana.

${ }^{30}$ Datos pormenorizados en Vicente Sánchez (2006: 115, n. 79).

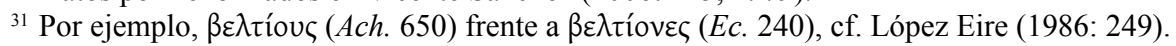

${ }^{32}$ Vicente Sánchez (2006: 116).

${ }^{33}$ Una búsqueda mediante el $T L G$ da como resultado 10 ejemplos pertinentes de $\pi \lambda \lambda^{\prime} i ́ \omega v, 6$ de $\kappa \rho \varepsilon i ́ \tau \tau \omega \nu$,

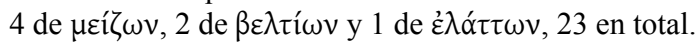

${ }_{34}$ Threatte (1996: 313s.); $\sigma \varphi \tilde{\omega} v \alpha \hat{\tau} \tilde{\omega} v$, etc. apenas aparecen tras esa fecha.

${ }^{35}$ Mayser I, 2, p. 63. 
aticistas. ${ }^{36}$ En Caritón es esto mismo lo que encontramos, ${ }^{37}$ y carece de importancia

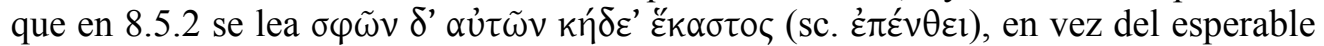
$\dot{\varepsilon} \alpha \cup \tau \tilde{\omega} v$, ya que este pasaje alude a $I l$. 19.302. Por lo tanto, con respecto a esta cuestión el novelista presenta formas áticas que también se emplean en la koiné.

La segunda cuestión que merece ser tratada es la sustitución de los pronombres reflexivos de 1. ${ }^{\mathrm{a}}$ y 2 . $^{\mathrm{a}}$ personas, tanto singular como plural, por el pronombre de 3 . $^{\mathrm{a}}$ persona. El uso del reflexivo de la 3. ${ }^{a}$ persona de plural en lugar de la 1.a y 2. ${ }^{a}$ está atestiguado tanto en inscripciones como en autores ya en el periodo clásico, ${ }^{38} \mathrm{y}$ es más frecuente en época tardía. ${ }^{39}$ Este uso se extiende del plural al singular. ${ }^{40}$ Los autores clásicos testimonian estos usos de formas diversas, que no responden a un patrón común. ${ }^{41}$

En Caritón encontramos $\dot{\varepsilon} \alpha \cup \tau \tilde{\omega} \vee 4$ veces en vez de la 1. ${ }^{a}$ pers. pl., (1.10.7, 7.1.11, $7.2 .4,8.2 .11)$, mientras que las combinaciones del pronombre personal y $\alpha \hat{\tau} \tau \tilde{\omega} v$ no aparecen. Con respecto al singular, se da el uso de $\dot{\varepsilon} \alpha u \tau o \tilde{v}$ como 2. a persona en tres ocasiones: $2.5 .5,6.2 .5,6.3 .8$. Se trata de casos en los que filólogos y editores han creído apreciar corrupciones textuales. Así, Reardon (2004) corrige el texto del ms. ${ }^{42}$ en los

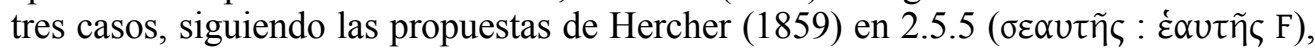

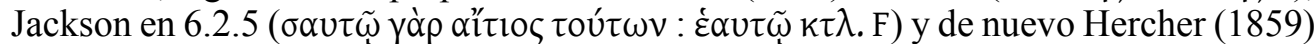

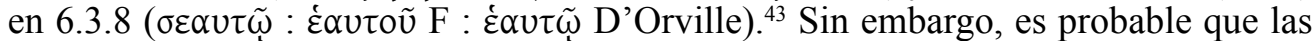
lecciones transmitidas por $\mathrm{F}$ respondan a un uso lingüístico genuino de Caritón. En primer lugar, son tres los pasajes; en segundo lugar, se da el uso paralelo (4 veces) de $\dot{\varepsilon} \alpha \cup \tau \tilde{\omega} \nu$ como 1. ${ }^{a}$ pers. pl.; por último, autores tardíos, incluso aticistas, atestiguan de maneras diversas la irrupción del reflexivo de 3. ${ }^{a}$ persona en el ámbito de las dos primeras, ya sea en singular o plural. ${ }^{44}$

El tercer y último problema se refiere a las formas, $\dot{\varepsilon} \alpha u \tau o \tilde{v}$, etc., y $\sigma \varepsilon \alpha u \tau o \tilde{v}$, etc., que dan lugar en ático a formas contractas $\alpha \dot{u} \tau o \tilde{u}$, etc. y $\sigma \alpha u \tau o \tilde{v}$, etc. El asunto cuenta

${ }^{36}$ Por ejemplo, en Libanio: cf. López Eire (1991: 97).

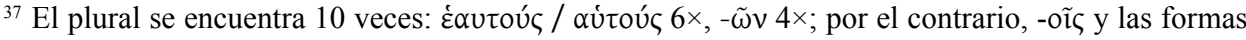
femeninas no aparecen.

${ }^{38} \mathrm{~K}$.-Bl. I, 599; hay ejemplos en los trágicos, Heródoto, Isócrates, etc.

${ }^{39}$ Mayser I, 2, p. 64; para el NT, cf. Bl.-D.-R. §64.1 y 283.

${ }^{40}$ Mayser, ibid.; Schmid, Atticismus I, 82, 228; IV, 69. En el NT es dudoso: B1.-D.-R. §64 ${ }_{1}$

${ }^{41}$ Ps.-Jenofonte emplea las formas de plural compuestas, pero a veces usa las formas en $\dot{\varepsilon} \alpha u \tau-$ para sg. y pl. (Caballero López 1997: 31 ss.; conclusiones en p. 35). El plural $\dot{\alpha} u \tau \tilde{\omega} v$ etc. aparece en Tucídides sustituyendo a las dos primeras personas (López Eire 1984: 259) y en Isócrates para la 1. a , además de usar la 3. ${ }^{a}$ sg. en vez de la 1. ${ }^{a}$ y 2. ${ }^{a}$. En general proporciona datos Vicente Sánchez (2006:119).

${ }^{42}$ Se trata del códice Florentinus Conv. Soppr. 627 (F en las ediciones, pero L en la de Molinié), que es codex unicus para el $95 \%$ de la novela, incluidos los tres casos anteriores. Sobre las ediciones, véase n. 3.

${ }^{43}$ La corrección de John Jackson a 6.2.5 no está publicada; es una de las correcciones realizadas por él en los márgenes de su propio ejemplar de la edición de Hirschig (1856), libro que está depositado en el Queen's College de Oxford y que fue utilizado por Goold (1995) y Reardon (2004) en sus ediciones; la cita Reardon (2004: ad loc.). En cuanto a 6.3.8, parece indudable que F contiene al menos una corrupción, ya que la sintaxis exige el dativo, de ahí que tanto D'Orville $\left(1783^{2}\right)$, en la editio princeps de la novela, como el propio Hercher (1859) propusieran este caso.

${ }^{44}$ Vicente Sánchez (2006: 119) aporta datos sobre Josefo, Luciano y Galeno; además, las Cartas de Temístocles revelan un uso idéntico al de Caritón en el singular (empleo de la 3. a persona por la 2.a , con 4 ejemplos, uno quizá corrupto), aunque no en el plural, ya que aquí esta obra sigue la norma ática (Vicente Sánchez 2006: 118). 
con la dificultad añadida de que durante la transmisión manuscrita puede haber habido corrupciones textuales en un sentido $u$ otro, dado el parecido de las formas. No obstante, dado que hay un número amplio de ejemplos, podemos pensar (sin tener la seguridad absoluta) que dicho fenómeno no afecta de manera decisiva a los resultados. Sobre la evolución de estas formas digamos de manera muy breve ${ }^{45}$ que las bisilábicas, que coexisten con las formas trisilábicas en ático, van siendo sustituidas por estas en época helenística. ${ }^{46}$ En los papiros ya no están atestiguadas las formas contractas en el siglo i a.C., y el NT las desconoce. ${ }^{47}$ En Caritón encontramos ambas series: غ́ $\alpha u \tau o \tilde{v}$,

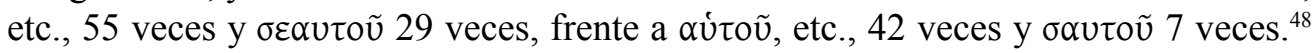
En total, 84 ejemplos de la serie trisilábica por 49 de la contracta. Este resultado no se diferencia mucho del que encontramos en prosistas clásicos como Tucídides o Jenofonte ${ }^{49}$ y se parece mucho también al de una obra quizá contemporánea de Caritón como las Cartas de Temístocles. ${ }^{50}$ El hecho de que en Caritón sea mayoritaria la forma trisilábica frente a la contracta parece cuadrar dentro de la evolución antes trazada. Junto a esto, la presencia todavía abundante de ejemplos de la forma contracta muestra que Caritón se hace eco de una forma ática presente sobre todo en la literatura. Tendríamos un nuevo ejemplo de influencia literaria en la prosa de Caritón.

Todo ello lleva a pensar que, en las tres cuestiones analizadas en este apartado, Caritón se hace eco de usos lingüísticos existentes ya en el dialecto y los autores áticos, que después continúan vigentes en la koiné y en diversos autores tardíos. No es posible, por tanto, hablar de aticismo de Caritón en lo tocante a estos puntos. ${ }^{51}$

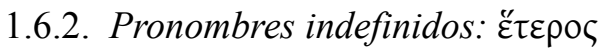

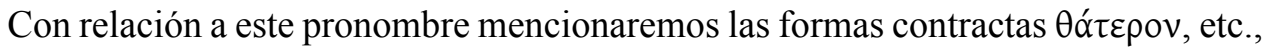
que son propias del ático ${ }^{52}$ y que aparecen normalmente en escritores tanto clásicos ${ }^{53}$ como tardíos.

${ }^{45}$ Un resumen informativo, con datos de inscripciones áticas y papiros, en Vela Tejada (1991: 132-133).

${ }^{46}$ En las inscripciones áticas (téngase en cuenta que la $1 .^{\mathrm{a}}$ y $2 .^{\mathrm{a}}$ personas son muy raras, y que el singular de la 3. ${ }^{\mathrm{a}}$ está muy poco atestiguado) las formas contractas son ya menos frecuentes desde el siglo v, con un único ejemplo, pero muy inseguro (Threatte 1996: 315). Hay más ejemplos después del 400 a.C., y tras el 300 a.C. se incrementa algo más su número, pero están siempre en minoría (ibidem).

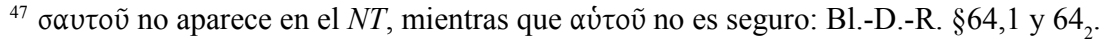

${ }^{48}$ Recuento hecho sobre los datos del $T L G$, que usa la edición de Reardon (2004).

${ }^{49}$ Un recuento en el $T L G$ da como resultado aproximado una proporción de 3 a 1 a favor de $\dot{\varepsilon} \alpha u \tau o \tilde{v}$ en Tucídides (de $\sigma \varepsilon \alpha u \tau o \tilde{v}$ hay un único ejemplo), y de 2 a 1 en Jenofonte. En cambio, en Jenofonte cam-

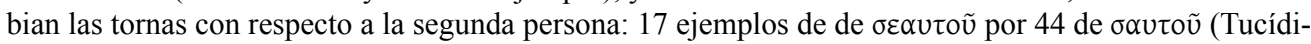

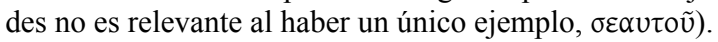

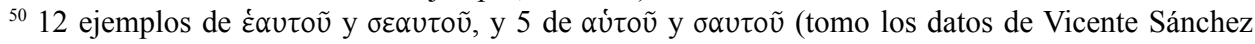
2006: 119).

${ }^{51}$ Por el contrario, otros autores, como es el caso de los aticistas Eliano y Aristides, sí se ciñen al uso normativo del ático (Vicente Sánchez 2006: 119).

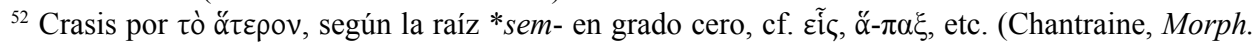
$\S 140)$.

${ }^{53}$ Por ejemplo, 4 veces en Tucídides y 6 en Jenofonte (datos del $T L G$ ). 
Puede ser de interés el siguiente hecho. Tardíamente surgen formas «incorrectas»

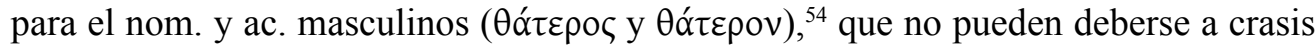

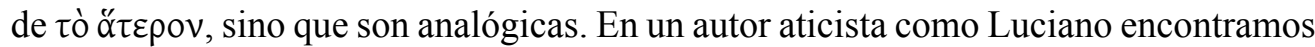

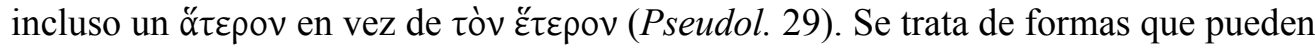
aparecer o no en autores aticistas, y que Schmid considera rasgos de baja koiné que se han introducido en su lengua. ${ }^{55}$ Sin embargo, el hecho es que no están atestiguadas en los papiros ni en el $N T .^{56}$

El hecho es que en Caritón estas formas quedan limitadas a cuatro ejemplos

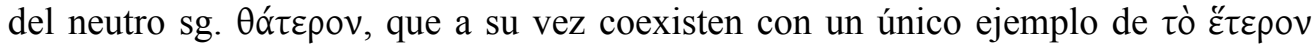
(2.10.8), ${ }^{57}$ una circunstancia que se da también en autores clásicos. ${ }^{58}$ Por todo ello parece que Caritón muestra un panorama acorde con el aticismo.

\subsubsection{Pronombres relativos y relativo-indefinidos; adverbios relativos}

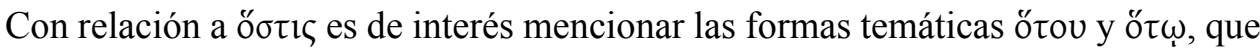
predominan en la prosa clásica hasta el siglo IV a.C. y reaparecen de manera ocasional en la koiné.$^{59} \mathrm{Su}$ presencia es, por tanto, indicio de influencia clasicista, y se dan en autores tardíos, incluidos los de la Segunda Sofística. ${ }^{60}$ Sin embargo, en Caritón solo

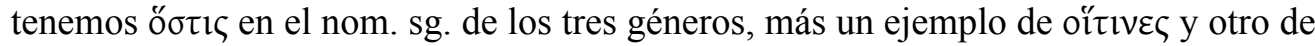
$\tilde{\omega} v \tau i v \omega v$.

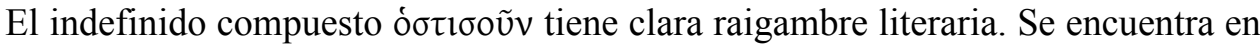
los prosistas áticos, pero apenas está atestiguado en los papiros y el NT lo desconoce. ${ }^{61}$

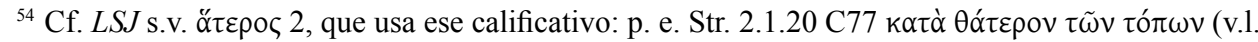

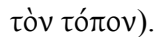

${ }^{55}$ Las usan Polemón, Dion Crisóstomo y Luciano; se limitan a las «correctas» Aristides, Eliano y Filóstrato (Schmid, Atticismus IV, 587, con los datos sobre los autores en I, 48, 85, 227; II, 19; III, 295; IV, 473). Schmid no lo afirma, pero creo que se trata a todas luces de hiperaticismos.

${ }^{56}$ Cf. Mayser I, 2, p. 70 y II, 2, p. 88-90; B1.-D.-R. $\$ 306,1$ y $306_{1}$.

${ }^{57}$ Tres de estos ejemplos $(2.10 .4,3.5 .6,5.6 .9)$ muestran lo que se podría considerar una expresión

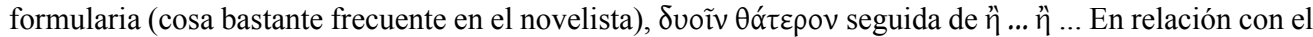

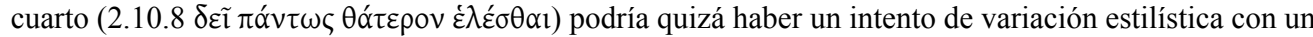

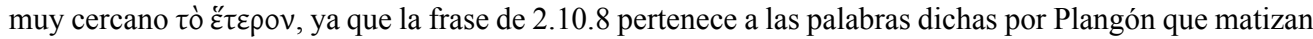

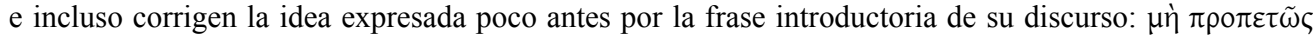

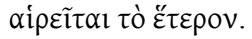

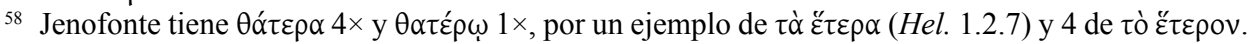

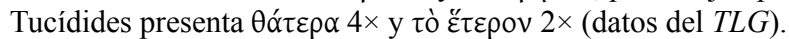

${ }^{59}$ En Heródoto y Ps.-Jenofonte solo aparecen las formas temáticas; en Tucídides ocurre igual, con un

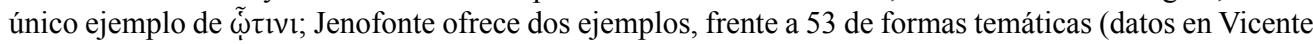
Sánchez 2006: 120-121). Según Threatte (1996: 333), las inscripciones áticas solo atestiguan ötov y ö $\omega$, con un único ejemplo dudoso de $\tilde{\omega} \tau \imath v v$.

${ }^{60}$ Las Cartas de Temístocles tienen solo dos ejemplos, 12.5 ö $\omega$ y 15.3 ötov (Vicente Sánchez 2006: 120-121).

${ }^{61}$ Tucídides lo usa una vez, Jenofonte 4 veces, en tres obras diferentes (datos del $T L G$ ); para los

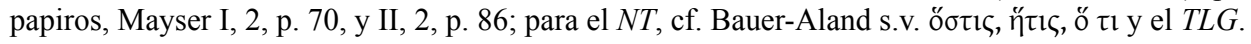


Sin embargo, en autores tardíos aparece con normalidad. ${ }^{62}$ Caritón escribe en 7.4.3

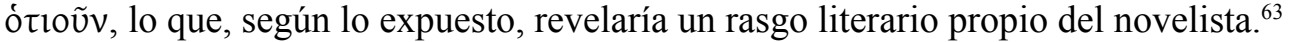

El adverbio relativo örtov alterna con oũ en ático, estando atestiguados ambos en las inscripciones, ${ }^{64}$ mientras que el primero es más habitual en los textos literarios. ${ }^{65}$ Posteriormente, ambos seguirán apareciendo en los papiros y en el $N T .{ }^{66}$ Caritón utiliza ö́rov, ${ }^{67}$ lo que no debe extrañar según lo dicho antes.

Quizá más interesante para este estudio es el valor sintáctico que tiene örov, ya que en los escritores aticistas suele tener valor causal. ${ }^{68} \mathrm{Sin}$ embargo, de los trece ejemplos que presenta Caritón tiene valor local en doce, ${ }^{69} \mathrm{y}$ solo en 5.6.10 presenta un valor diferente, que se puede considerar temporal-causal. ${ }^{70}$ Por lo tanto, lo que hay en Caritón es el valor más habitual del adverbio en griego y más en concreto en la koiné. Por otro lado, el valor temporal está atestiguado en la literatura, ${ }^{71}$ por ejemplo A., Cho.

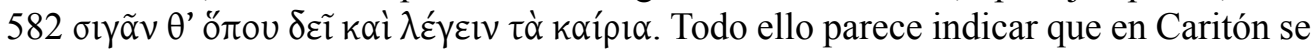
da el uso típico de la koiné helenística, que ya viene del ático, y que el único caso que se sale de este uso puede atribuirse a influencia literaria.

En resumen, a la vista de los datos de este apartado puede afirmarse que en Caritón los pronombres y adverbios relativos muestran en su conjunto un griego propio de la koiné que no presenta rasgos aticistas estrictos, y sí rasgos que responden a un influjo literario ático.

\subsubsection{Pronombres numerales (cardinales): $\alpha^{\prime} \mu \varphi \omega$ y $\alpha \dot{\alpha} \mu \operatorname{có}_{\varepsilon} \rho \mathrm{\imath}$}

El dual $\alpha^{\alpha} \mu \varphi \omega$ va dejando su lugar en griego a $\alpha \mu \varphi o ́ \tau \varepsilon \rho o 1,{ }^{72}$ que es el término usado tanto en los papiros como en el $N T .^{73}$ Sin embargo, el gen. y dat. $\alpha \mu \varphi$ oĩv se conserva en algunos papiros tardíos, ${ }^{74}$ y puede aparecer solo o acompañando a un nombre dual en

${ }^{62}$ Por ejemplo, 20 apariciones en Plutarco y 10 en Luciano (datos del TLG).

${ }^{63}$ Coincide exactamente con las Cartas de Temistocles, obra que atestigua este mismo pronombre también una vez: 6.8 ó $\tau$ Ioṽv (cf. Vicente Sánchez 2006: 122).

${ }^{64}$ En las que oṽ es muy frecuente (Threatte 1996: 408).

${ }^{65}$ En Jenofonte, por ejemplo, örov aparece 93 veces, oũ 33 (datos de tres obras, Helénicas, Anábasis y Ciropedia, tomados de Vicente Sánchez 2006:121-122).

${ }^{66}$ Mayser II, 1, p. 76-77; B1.-D.-R. \$293,1 y 293 : en el NT es mayoritario ö ejemplos).

${ }^{67}$ En 13 ocasiones según la edición de Reardon (2004), pero hay que advertir que 1.11.5 es conjetura de Reiske, ya que F lee öđol. En cuanto a oũ, que está 9 veces, se trata del gen. del pronombre relativo,

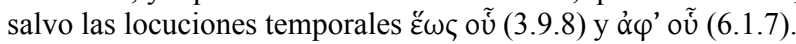

${ }^{68}$ Sobre este valor, cf. LSJ s.v. II, 2; Schmid, Atticismus I, 129; II, 134-135; III, 142; IV, 208.

${ }^{69} 1.11 .5$ (pero véase $n .67$ ), 2.4.7, 2.4.10, 3.2.16, 3.6.2, 3.9.12, 3.10.3, 5.1.5, 5.1.6, 5.2.8, 8.2.12, 8.8.15.

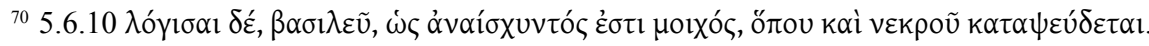

${ }^{71}$ LSJ s.v. II, 1.

${ }^{72}$ Schwyzer I, 589; Chantraine, Morph. $§ 163$.

${ }_{73}$ Mayser I, 2, p. 70 y II, 2, p. 93-94; B1.-D.-R. $\$ 64,6$ y $64_{10}$.

${ }^{74} \mathrm{Si}$ bien son muy escasos; además de varios ejemplos de época bizantina, Gignac (1981: 190) cita uno del siglo II y dos del III. 
un autor como Polibio. ${ }^{75}$ Por lo demás, el dual seguirá apareciendo en autores tardíos como un rasgo de aticismo. ${ }^{76}$ Esto podría suceder en el caso de Caritón, ya que, frente a 20 apariciones de $\alpha \mu \varphi$ ó $\varepsilon \rho \circ \varsigma$, utiliza dos veces $\alpha \mu \varphi$ ĩv en dativo (4.4.2 у 5.10.6, cf. 1.12.10). En todo caso, y a la vista de los números, se trata de una forma escasamente utilizada, ya que, junto a estos dos dativos, Caritón emplea ỏ $\mu \varphi$ óćpoıৎ 4 veces $(1.1 .18,1.13 .6,7.5 .7,7.6 .2)$. Parece probable, por tanto, que la presencia de $\alpha \mu \varphi$ ĩv sea un rasgo literario, un arcaísmo usado por ciertos escritores y por los autores de algunos documentos papiráceos para dar un barniz más «literario» a sus escritos. ${ }^{77}$

\section{7. -í deíctica}

Caritón no usa nunca la -í deíctica propia del ático, ni siquiera en el adverbio vuví, única palabra que posee esta forma deíctica en el $N T .^{78}$ Tenemos, pues, un caso claro de ausencia de un rasgo aticista, rasgo, que, en cambio, está presente en todos los demás novelistas. ${ }^{79}$

\subsection{Sufijos $-\theta \varepsilon v,-\theta \mathrm{l},-\delta \varepsilon$}

Se trata de sufijos típicos de la prosa ática, que indican procedencia, lugar en donde y lugar a donde, respectivamente. Se pueden añadir tanto a adverbios como, en el caso de $-\theta \varepsilon v$ y $-\delta \varepsilon$, a nombres propios de lugar. Estos dos coinciden en su evolución, ya que en los papiros quedan restringidos a los adverbios, igual que sucede en el $N T$, mientras que posteriormente los autores aticistas los recuperan para acompañar también a topónimos.

En Caritón encontramos ambos sufijos, pero solo aplicados a adverbios, ${ }^{80}$ nunca a

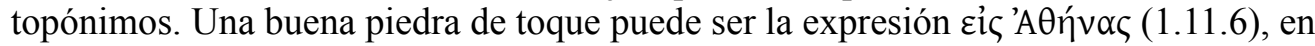
lugar de la típica expresión ática 'A $\theta \eta \dot{v} \vee \not \zeta \varepsilon$, que está presente ya en las inscripciones áticas y es muy frecuente en autores clásicos y tardíos. ${ }^{81}$ En consecuencia, Caritón no registra un rasgo claro de lengua aticista.

${ }^{75}$ En efecto, en Polibio solo han sobrevivido las formas de dual en -olv (Foucault 1972: 69), lo que se ha explicado (Cuny, citado por Foucault) porque estas (junto con las formas en - $\alpha \mathrm{lv}$ ) eran formas antiguas y características que no corrían el riesgo de confundirse con otras, lo que las convertía en las más resistentes.

76 Schmid, Atticismus I, 87-88; II, 35-36; III, 46 ss., 611.

${ }^{77}$ Puede ser este el lugar indicado para señalar que Caritón usa por dos veces $(2.10 .4,3.5 .6)$ la forma

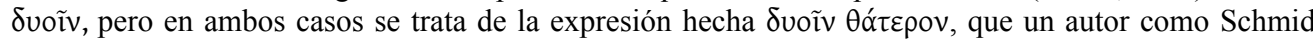
(Atticismus II, 35) considera fórmula excluible del elenco de aticismos. Todo ello apunta a que $\alpha \mu \varphi$ ĩ $v$ y ¿voĩv son términos literarios de uso muy ocasional y, por así decir, formas artificiales en un autor que, por lo demás, no usa en absoluto el dual.

${ }^{78}$ Bl.-D.-R. $\$ 64_{4}$.

${ }^{79}$ Datos en Papanikolaou (1973: 92): Jenofonte 5× (de ellas, 4× vuví), Longo $2 \times$, Aquiles Tacio $3 \times$, Heliodoro $9 \times(5 \times$ vuví $)$.

${ }^{80}$ Es muy mayoritario el sufijo - $\theta \varepsilon v$, formando parte de diferentes adverbios ( $\pi \alpha \nu \tau \alpha \chi \chi \theta \varepsilon v, \pi o ́ \rho \rho \omega \theta \varepsilon v$, $\alpha^{\prime} v \omega \theta \varepsilon v$, etc.), mientras que $-\delta \varepsilon$ solo está en $\varepsilon \dot{v} \theta \alpha ́ \alpha \varepsilon(13 \times, 1.10 .7$, etc.).

${ }^{81}$ Para las inscripciones, cf. Threatte (1996: 405); entre los autores clásicos (datos del TLG), cf. por ejemplo Jenofonte 9×, Ps.-Jenofonte $2 \times$ (1.16 y 1.18, cf. Caballero López 1997: 28); entre los tardíos,

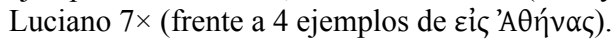




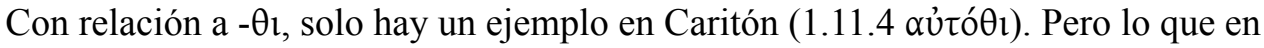
principio es un rasgo aticista queda en entredicho por dos circunstancias: $\alpha u ̉ \tau o ́ \theta t ~ a p a-$ rece en la koiné, según el testimonio de los papiros; ${ }^{82}$ Caritón no usa otros adverbios en $-\theta \mathrm{l}$ que, sin embargo, están presentes en otros autores tardíos. ${ }^{83}$

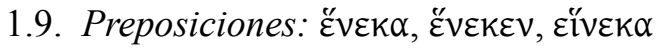

La preposición ह̌ $v \varepsilon \kappa \alpha$ es la que aparece en las inscripciones áticas, ${ }^{84}$ mientras que

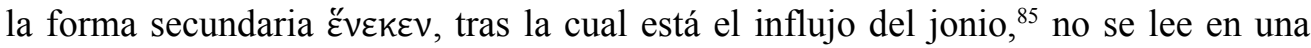
inscripción datada con seguridad hasta $302 / 1$, y sin duda no está documentada antes

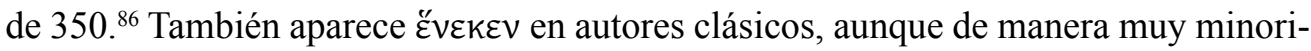

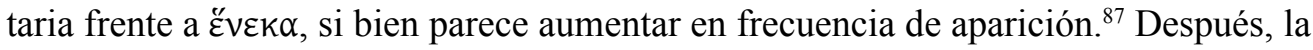

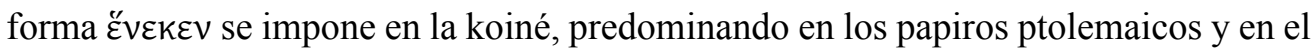

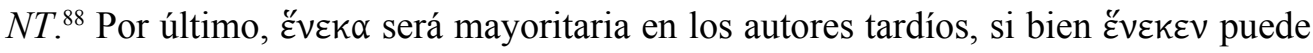

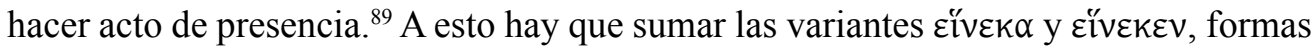
épicas, jónicas y poéticas, tributarias de la tradición literaria. ${ }^{90}$

${ }^{82}$ Es el único adverbio en - $\theta$ t: Mayser I, 3, p. 119 (también 206); II, 2, p. 169, 176. Más tarde lo retoman los aticistas: Schmid, Atticismus V, 81 y esp. I, 112.

${ }^{83}$ Las Cartas de Temistocles muestran una situación bastante diferente a la de Caritón, con 4 ejemplos de $\alpha u ̉ \tau o ́ \theta t$, pero también uno de ớ $\lambda \lambda_{\circ} \theta \mathrm{r}$. Lo más interesante puede ser que esta obra tiene también un ejemplo de $\dot{\varepsilon} \kappa \varepsilon \tilde{\theta} \theta \mathrm{l}$ (20.3), un adverbio no usado por la prosa ática y que, por tanto, podría ser indicativo de

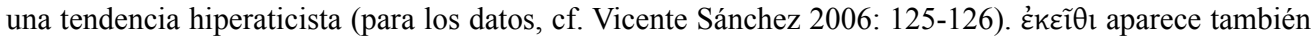

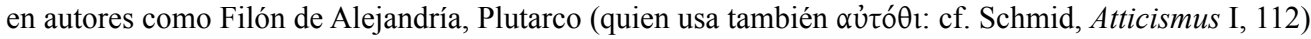
y Eliano (cf. Vicente Sánchez 2006: 126).

${ }^{84}$ Threatte (1996: 660).

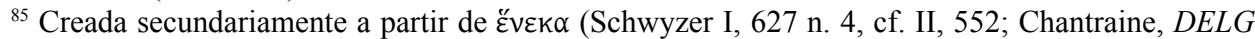

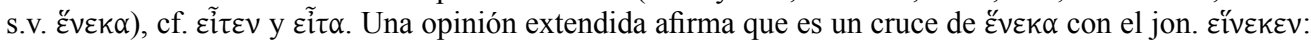
B1.-D.-R. §30 ${ }_{5}$; López Eire (1981: 40); Vela Tejada (1991:107), quien cita a J.H. Moulton, A Grammar of New Testament Greek (Edimburgo, 1960), vol. II, 67.

${ }^{86}$ Threatte (1996: 660; comentario de algún caso problemático en p. 661); después, desde comienzos del siglo III a.C. hasta época romana tardía, tanto una como otra son frecuentes, e incluso ambas aparecen en algunos textos (ibidem).

${ }^{87}$ Según el $T L G$ y en general las ediciones, Tucídides tiene un solo ejemplo (6.2.6, ante consonan-

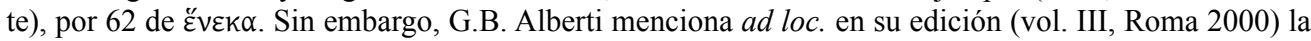

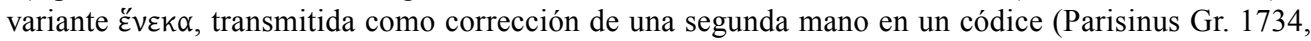
saec. XIV in.) y, lo que es más interesante, como lectura del P. Bodmer XXVII, de los siglos III-IV d.C. Se

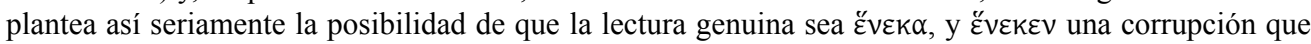

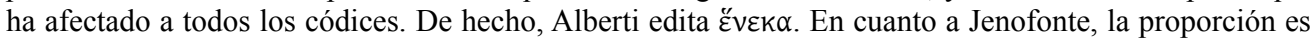

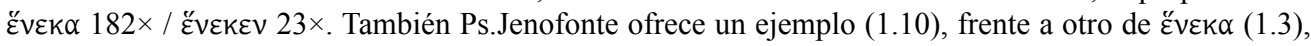
cf. la discusión en Caballero López (1997: 18).

${ }^{88}$ Mayser I, 1, p. 12; BL.-D.-R. $\$ 30_{5} ; 216_{1}$.

${ }^{89}$ Como ejemplo citaremos a Luciano (incluidas las obras espurias), donde tenemos ह̌vek $136 \times /$

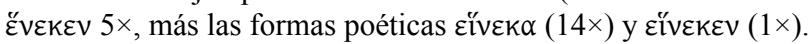

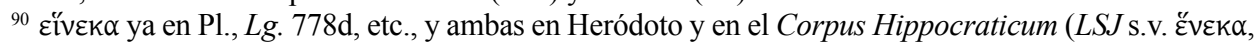

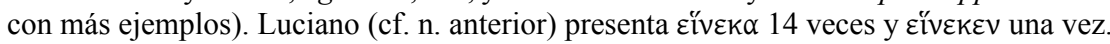


Es necesario aludir a una faceta del problema, mencionada por algunos autores, ${ }^{91}$ la

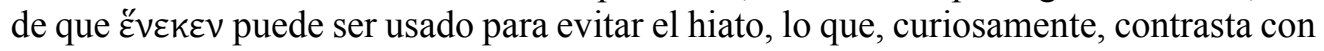

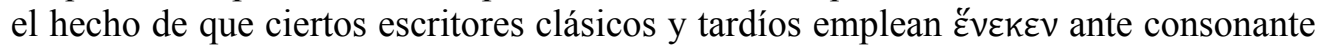

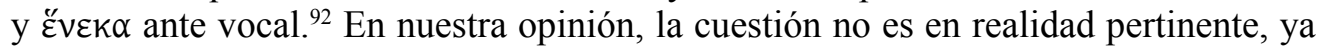
que la vocal final de Ěveka se elide ante palabra que comience por vocal, y al haber elisión no se produce el hiato. Por lo tanto, el autor antiguo se veía en realidad ante una opción que podemos considerar de estilo, la de usar un término de raigambre ática

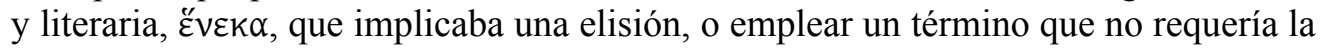

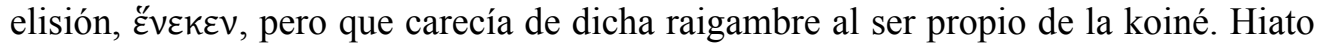
no había en ningún caso.

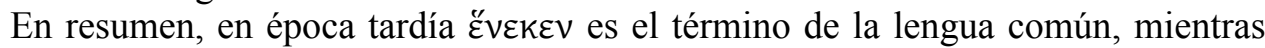

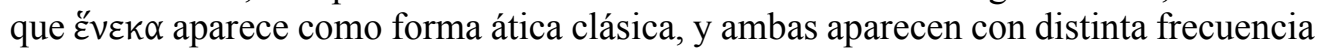
según épocas y fuentes.

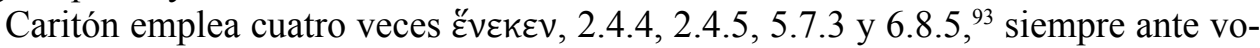

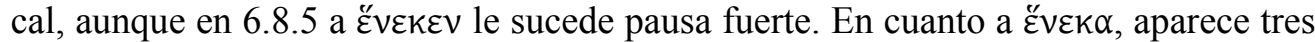

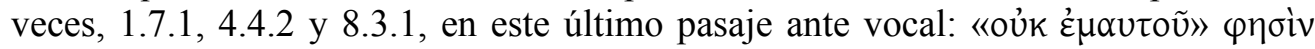

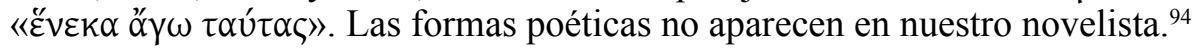

En consecuencia, y hecha la salvedad de que no es imposible la existencia de una corrupción textual, lo que podría cambiar el panorama dada la escasez de ejemplos, los datos avalan que no nos hallamos ante un caso de aticismo, ya que Caritón usa ambos términos de manera similar, tanto el término propio de la koiné como el clásico. Tendríamos, por tanto, un ejemplo de prosa «clasicista», anterior o al menos ajena a los rigores del aticismo.

\section{MORFOLOGÍA VERBAL}

\subsection{Aumento}

\subsubsection{El aumento de $\theta \dot{\varepsilon} \lambda \omega$ / $\varepsilon \theta \varepsilon \dot{\lambda} \lambda \omega$}

No tocaremos aquí el problema del doblete verbal $\dot{\varepsilon} \theta \dot{\varepsilon} \lambda \omega / \theta \varepsilon ́ \lambda \omega .^{95}$ Solo nos referiremos brevemente a la cuestión del aumento, para señalar que las formas con $\eta \dot{\theta} \theta \lambda$ -

${ }^{91}$ Cf. el, por otra parte, completo análisis de Vicente Sánchez (2006: 127-129), quien plantea el problema en términos de presencia del hiato.

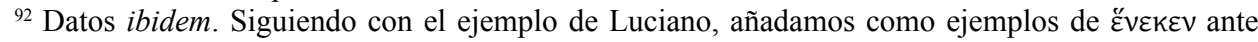

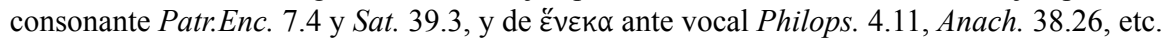

${ }^{93}$ En 2.4.5 se trata de un suplemento de Reiske (en D'Orville $1783^{2}$ ad loc.), que los editores consi-

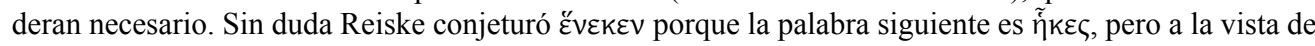
los ejemplos de $\check{\varepsilon} v \varepsilon \kappa \alpha$, y en concreto 8.3.1, esta forma habría sido también posible.

${ }^{94}$ Una obra como las Cartas de Temistocles, quizá contemporánea de Caritón, ofrece resultados

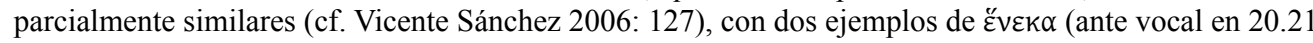
y ante pausa más vocal en 8.31) por uno de ĚvekEv (20.11, ante consonante). La diferencia fundamental

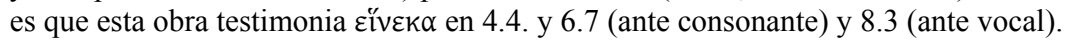

${ }^{95}$ Sobre este aspecto de la lengua de Caritón, cf. Sanz Morales (2014: 295). 
(correspondientes a $\dot{\varepsilon} \theta \varepsilon ́ \lambda \omega$, como es natural) son casi unánimes a lo largo de la historia de la lengua griega, tanto en la koiné como en los autores literarios. Hay que señalar, no obstante, que existe también la posibilidad del aumento silábico, $\dot{\varepsilon} \theta \varepsilon \lambda-.^{96}$

Caritón presenta un panorama común en la koiné y en los autores tardíos, consis-

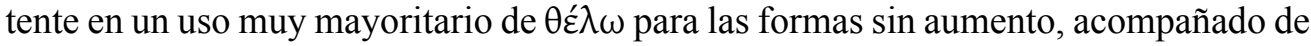

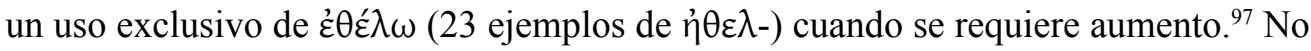
hay, pues, influencia de la lengua poética, sino una situación de «normalidad», según la cual el rasgo analizado no caracteriza de forma especial la lengua caritonea.

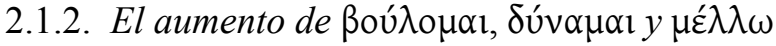

Estos verbos pueden presentar, junto al aumento «normal» en $\dot{\varepsilon}$-, un aumento en $\eta \dot{\eta}$-, que aparece ya en las inscripciones áticas a partir del 300 y se explica por analogía con el verbo, de sentido cercano, $\theta \dot{\varepsilon} \lambda \omega$ / $\dot{\varepsilon} \theta \dot{\varepsilon} \lambda \omega$, en el que el imperfecto quirió la apariencia de una forma verbal con aumento largo. ${ }^{98} \mathrm{En}$ los autores literarios

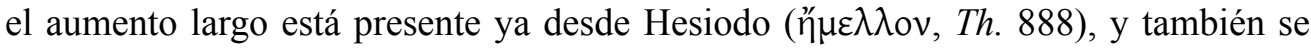
documenta en los géneros dramáticos (E., Hel. 752 y Ar., Ec. 597). La consolidación del doblete en la poesía sin duda resultó favorecida por las necesidades métricas. En cuanto a la prosa clásica, se suelen aducir ejemplos en Tucídides $(2.2 .3,6.79 .3)$ y Ps.Jenofonte $2.14,{ }^{99}$ pero se trata de formas al menos dudosas, si nos atenemos a los mss., y lo cierto es que los editores suelen preferir las formas con $\dot{\varepsilon}$-. Un ejemplo ya

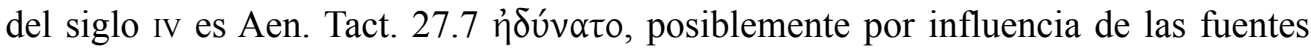
literarias. ${ }^{100}$ El hecho es que en la prosa clásica las formas con $\eta$ - se revelan, si no inexistentes, al menos muy minoritarias. ${ }^{101}$

Después, el aumento largo parece haberse impuesto en la lengua común, ya que

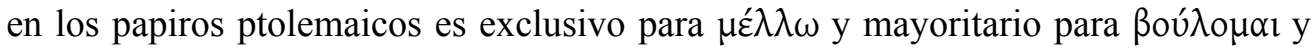

${ }^{96}$ Son típicas de la épica y lírica: $\varepsilon^{\prime} \theta \varepsilon \lambda$ ov Il.6.165, 636, Thgn. 606, B. 10.73; $\varepsilon \dot{\varepsilon} \lambda \eta \emptyset \sigma \alpha$ Il. 18.396 (cf. $L S J$ s.v. $\dot{\varepsilon} \theta \dot{\lambda} \lambda \omega$ ).

${ }^{97}$ Sanz Morales (2014: 295).

${ }^{98}$ Chantraine, Morph. $§ 356$. Sobre las inscripciones áticas, Threatte (1996: 474) precisa que el aumento en $\dot{\varepsilon}$ - no se documenta después de la década del 330 , mientras que el aumento en $\eta$ - aparece por vez primera alrededor del 350 y es normal en lo sucesivo.

${ }_{99}$ Así, Vela Tejada (1991: 139) y Caballero López (1997: 40, con discusión de la discrepancia de los

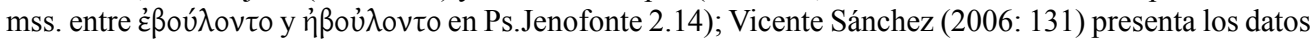
con mayor prudencia.

${ }^{100}$ Es la explicación de Vela Tejada (1991: 139). Pero es preciso indicar que el ejemplo no es del todo seguro, ya que el texto se basa en un codex unicus, siendo los cuatro códices restantes apógrafos sin valor.

${ }^{101}$ Una búsqueda por medio del $T L G$ en la obra de Platón y Jenofonte da como resultado que el pri-

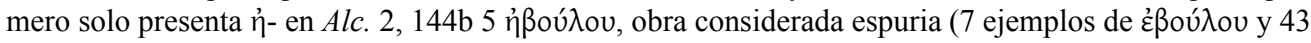

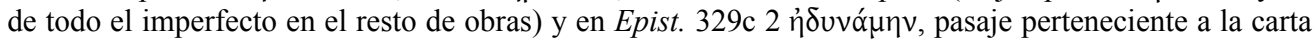

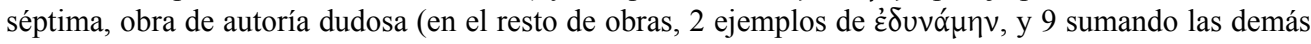
personas). En cuanto a Jenofonte, sus obras no ofrecen formas con aumento largo en ninguno de los tres verbos. 
$\delta u ́ v \alpha \mu \alpha{ }_{1}{ }^{102}$ Por último, el $N T$ documenta ambos tipos de aumento para $\delta u ́ v \alpha \mu \alpha l$ y

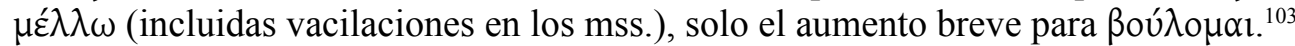

En cuanto a los autores tardíos, la casuística es muy amplia, ${ }^{104}$ pero una posible

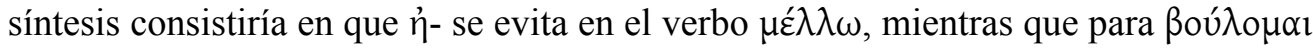
y $\delta u ́ v \alpha \mu \alpha ı$ la flexibilidad es mayor. En todo caso, el rigor aticista será directamente proporcional al predominio del aumento en $\dot{\varepsilon}$ -

Caritón coincide con varios autores aticistas en no admitir el aumento largo en el

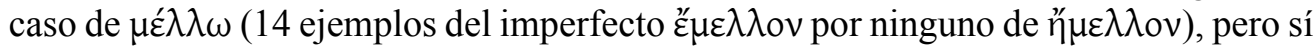

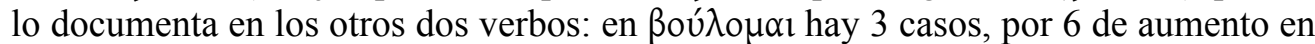
$\dot{\varepsilon}-$, y en $\delta u ́ v \alpha \mu \alpha l$ son 9 los ejemplos, por 11 de $\dot{\varepsilon}$-. Por tanto, ambos aumentos presentan una situación equilibrada en estos dos verbos, a lo que hay que sumar la existencia de

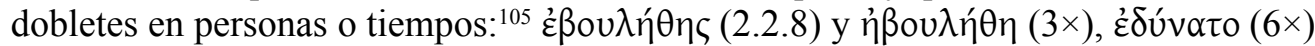

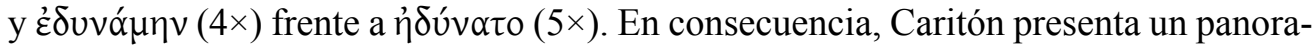
ma que podríamos catalogar de híbrido entre formas propias de la koiné y formas que remiten a la prosa clásica ática. ${ }^{106}$

\subsection{Desinencias}

\subsection{1. oĩ $\sigma \theta \alpha /$ oĩ $\delta \alpha \varsigma$}

La segunda persona del sg. de oĩ $\delta \alpha$ es en ático oĩ $\sigma \theta \alpha,{ }^{107}$ mientras que la correspondiente forma jonia es oĩ $\delta \alpha{ }^{108}$ Esta es la forma que acoge la koiné, y la única atestiguada por los papiros ptolemaicos y el NT. ${ }^{109}$

En autores aticistas, aunque oĩ $\delta \alpha \varsigma$ puede hacer acto de presencia, lo normal es oĩ $\sigma \theta \alpha$, como es lógico por ser esta la forma ática. Así, en autores como Dionisio de Halicarnaso, Dion Crisóstomo o Luciano predomina oĩ $\sigma \alpha \alpha$, mientras que en Aristides es exclusiva. ${ }^{110}$

${ }^{102}$ Mayser I, 2, pp. 93-94.

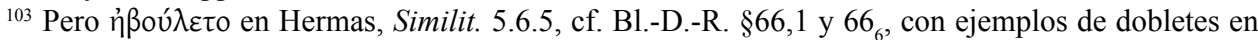
$\delta u ́ v \alpha \mu \alpha ı$ y $\mu \varepsilon ́ \lambda \lambda \omega$.

${ }^{104}$ Schmid, Atticismus I, 229; II, 22; III, 33; IV, 28 y 590 (conclusión). Hay también un útil resumen en Vicente Sánchez (2006: 132).

${ }^{105}$ Es decir, no hay una distribución de los dos aumentos según los diferentes tiempos: la unanimidad

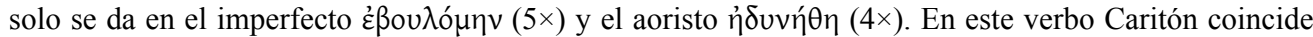

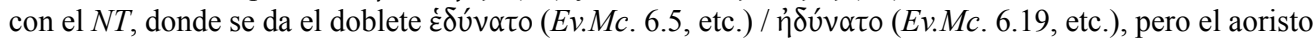

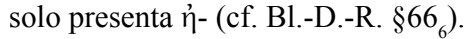

${ }^{106}$ Un uso más estricto de las formas áticas se da en las Cartas de Temístocles, obra que presenta

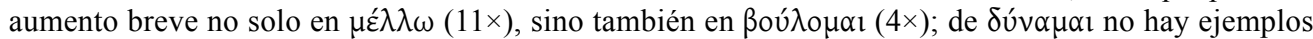
(cf. Vicente Sánchez 2006: 132).

${ }^{107}$ Ya aparece en Homero, Il. 1.365, etc.; después se creará oĩ $\sigma \theta \alpha \varsigma$, forma híbrida de oĩ $\sigma \theta \alpha$ y oĩ $\delta \alpha \varsigma$, atestiguada en Cratino y Menandro (Chantraine, Morph. §343).

${ }^{108}$ También en Heródoto; cf. Chantraine, Morph. §217.

${ }^{109}$ Mayser I, 2, p. 130; B1.-D.-R. $\$ 99,2$.

${ }^{110}$ Ofrece los datos Vicente Sánchez (2006: 133). Señala que en las Cartas de Temistocles aparecen las dos formas, además de la poética oĩo $\theta \alpha$. 
En este caso, el panorama observable en Caritón es muy claro, ya que no se documenta oĩ $\sigma \theta \alpha$, y solo se encuentra la forma oĩ $\delta \alpha \varsigma$ en las seis ocasiones en que aparece esta segunda persona. ${ }^{111}$ Es de interés señalar que en 2.4.7 el P.Oxy. 2948 confirma la forma oĩ $\delta$ 次 de $F,{ }^{112}$ con lo que poseemos un segundo testimonio de esta forma; es un testimonio antiguo, de fines del siglo II. Tenemos, pues, un ejemplo claro de término de koiné, sin que la obra de Caritón ofrezca huella alguna de aticismo. ${ }^{113}$

\subsection{2. $\tilde{\eta} \sigma \theta \alpha / \tilde{\eta} \varsigma$}

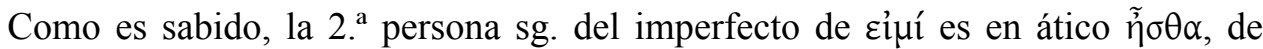
manera muy mayoritaria tanto en las inscripciones como en la literatura, frente a la forma jónica $\tilde{\eta} \varsigma$. Sin embargo, en la koiné se impone esta última, que se convierte en hegemónica en el $N T .{ }^{114}$ Más tarde, los autores aticistas utilizarán por lo general el esperable $\tilde{\eta} \sigma \theta \alpha$, aunque ejemplos de $\tilde{\eta} \varsigma$ pueden encontrarse también. ${ }^{115}$

En el caso de Caritón, los únicos dos ejemplos lo son de $\tilde{n} \varsigma(6.2 .6,6.2 .9)$. Este uso lingüístico coincide con el anteriormente mencionado (oî̃ $\delta \alpha \varsigma, \mathrm{y}$ no oĩ $\sigma \theta \alpha$ ), y revela de nuevo el predominio de la koiné. ${ }^{116}$

\subsubsection{Segunda persona sg., voz media}

Esta segunda persona puede presentar dos terminaciones, $-\eta$ y $-\varepsilon l$, siendo la primera de ellas la predominante en época clásica. Sin embargo, en los verbos temáticos, si bien encontramos en ático un indicativo del tipo $\varphi \varepsilon ́ p \eta$ (contracción tras * ${ }^{\mathrm{h}}$ eresai > $\varphi \varepsilon ́ p \varepsilon \alpha$ ), a partir del siglo IV aparece $\varphi \varepsilon^{\prime} \rho \varepsilon l$. Esto último sucede de manera constante en las formas verbales ßoú $\lambda \varepsilon l$ y oľ́l. En los verbos atemáticos, y a pesar de algunas vacilaciones del jónico-ático, que puede presentar formas contractas, se restablece la $\sigma$ intervocálica. Con la koiné, la desinencia $-\sigma \alpha l$ tiende a extenderse y pasa a la conjugación temática, llegando a ser creadas formas como $\varphi \varepsilon ́ p \varepsilon \sigma \alpha 1 .{ }^{117} \mathrm{En}$ los autores tardíos

${ }^{111} 1.7 .2,1.12 .3,2.4 .7,3.4 .8,5.2 .5,7.6 .10$. En 1.7 .2 se trata de una conjetura obvia de D'Orville y Hercher; en su apógrafo de F copiado entre 1727 y 1728, Antonio Cocchi escribió oĩ $\delta$, ya que es lo que parece tener el ms. (cf. ed. Reardon 2004 ad loc.: «oî $\delta \alpha$ F ut videtur, sed lectio incerta»); Reiske propuso corregir con la forma aticista oĩ $\sigma \theta \alpha$.

${ }^{112}$ El papiro lee or $\delta$, que ya el editor del papiro consideró error de escriba: «P's reading here is a mistake» (Weinstein 1972: 14). F tiene oĩ $\delta$ ç.

${ }^{113}$ De los demás novelistas (no hay ejemplos en Longo), Jenofonte usa solo oĩ $\delta \alpha \varsigma$ ( $2 \times$, y oí $\delta \alpha \mu \varepsilon v$ en 5.3.14); también Aquiles Tacio, 9 veces, pero tiene un ejemplo de oĩo $\theta \alpha$ (7.6.3). Sin embargo, Heliodoro presenta solo oĩ $\sigma \theta \alpha, 7$ veces (cf. Papanikolaou 1973: 94-95).

${ }^{114}$ Bl.-D.-R. $§ 98_{1}$; en los papiros ptolemaicos no está atestiguada la 2. ${ }^{\text {a }}$ pers. sg. del imperfecto: Mayser I, 2, p. 127.

$115 \tilde{\eta} \sigma \theta \alpha$ es única en Dionisio de Halicarnaso, Aristides, Eliano, Filóstrato, y mayoritaria en Luciano (Schmid, Atticismus I, 231; IV, 35, 599) y en las Cartas de Temístocles, con 5 de 6 casos en esta obra (Vicente Sánchez 2006: 133-134). ஸे s predomina en Plutarco, según indica Weissenberger (1994: 35-36),

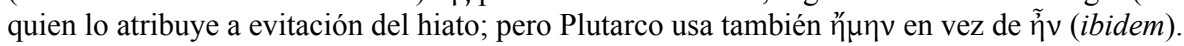

${ }^{116}$ Entre los novelistas se da la forma jónica en Jenofonte (4.6.7), pero tanto Aquiles Tacio (1.8.4) como Heliodoro (2.18.3) respetan la forma ática (Papanikolaou 1973: 95).

117 Para toda la cuestión, cf. Chantraine, Morph. \$344. 
no será extraño que aparezcan tanto - $\eta$ como - $\varepsilon$ l, incluso en el seno de la obra de cada autor, pero hay una tendencia a privilegiar $-\eta$, excepto en lo ya dicho con respecto a

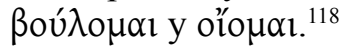

El principal problema que presenta esta cuestión es que, con el itacismo que afecta a las vocales largas de timbre $e$ en época helenística y el subsiguiente paso a $i,{ }^{119}$ la diferencia fonética entre $\eta$ ( $e$ larga abierta) y $\varepsilon$ ( $e$ larga cerrada) deja de existir. Ello significa que, en la práctica, el problema deja de ser fonológico y fonético para ser de escritura u ortográfico. La consecuencia de todo esto es que, como decíamos, en los mss. de muchos autores aparecen ambas grafías, y lo normal es que no haya criterios sólidos para determinar de manera unánime el carácter genuino de una. De ahí que, como opinan estudiosos del problema, parezca aconsejable no regularizar la lengua de un autor adoptando por sistema una de las dos terminaciones. La parte negativa, por así decir, es que resulta difícil obtener conclusiones determinantes en relación con el problema a partir de los datos existentes.

Sin embargo, es preciso al menos ofrecer estos datos, que en Caritón son los siguientes. Por lo general, aparece la desinencia $-\eta$ tanto en el presente como en el

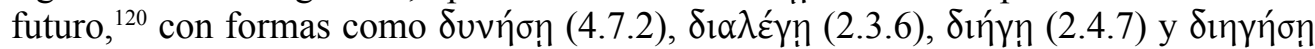

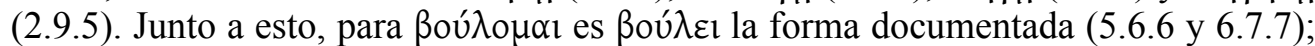
la 2. ${ }^{\text {a }}$ persona de oĺo $\mu \alpha$ no aparece. Se trata de una distribución normal en un autor tardío: Eliano o Filóstrato presentan panoramas muy semejantes, ${ }^{121}$ e igual sucede en una obra como las Cartas de Temístocles, quizá coetánea de Caritón. ${ }^{122}$

\subsubsection{Dual}

Como sucede con las desinencias nominales, en la koiné el dual desaparece de la flexión verbal. ${ }^{123}$ Más tarde, los autores aticistas lo recuperan, por lo que no es raro encontrar formas de dual en autores como Dion Crisóstomo, Luciano, Elio Aristides o Filóstrato. ${ }^{124} \mathrm{El}$ hecho de que en Caritón no aparezcan formas verbales en dual supone, por tanto, la ausencia de un claro rasgo aticista.

\subsection{Pluscuamperfecto}

El pluscuamperfecto posee una serie antigua

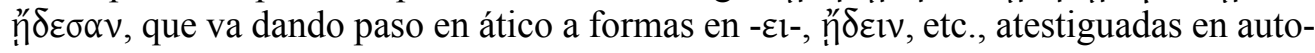

${ }^{118}$ Schmid, Atticismus I, 83, 230; II, 24; III, 30; IV, 26-27, 588-589; datos también en Vicente Sánchez (2006: 134).

${ }^{119}$ Lejeune, Phonétique §251.

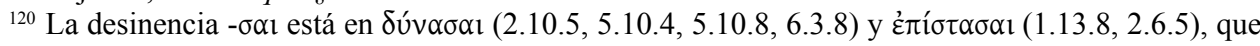
son también las formas normales en ático: cf. K.-Bl. II, 404 y 422, respectivamente.

${ }^{121}$ Schmid, Atticismus III, 30 (Eliano); IV, 26-27 (Filóstrato).

${ }^{122}$ Documenta en general la desinencia -n, pero ofrece tres ejemplos de $\beta$ oú $\lambda \varepsilon 1$ y uno de oľ́l (cf. Vicente Sánchez 2006: 135).

${ }^{123}$ Véase la n. 23.

${ }^{124}$ Schmid, Atticismus I, 87, 233; II, 35; III, 47-48; IV, 46-47. En los novelistas griegos hay un único

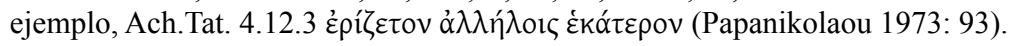


res del siglo IV. ${ }^{125}$ Esta serie tiende a imponerse con el paso del tiempo, de manera que en la koiné neotestamentaria es ya hegemónica. ${ }^{126}$ Sin embargo, en la 3. ${ }^{\mathrm{a}}$ pl. persiste la fluctuación entre $-\varepsilon \sigma \alpha \nu$ y - $\varepsilon l \sigma \alpha v$, como puede apreciarse en Polibio o Plutarco, así como en autores aticistas: Dion de Prusa presenta normalmente $-\varepsilon \sigma \alpha \nu$, pero Filóstrato tiene ambas. ${ }^{127}$

Caritón presenta las formas en - $\varepsilon$ l- en las tres personas del singular, pero la $3 .^{\mathrm{a}} \mathrm{pl}$. es n๊ $\delta \varepsilon \sigma \alpha v .{ }^{128}$ Se trata de la forma más genuinamente ática, lo que puede interpretarse como un intento de dar a la lengua un barniz ático o clásico. En ello coincide Caritón por completo con las Cartas de Temístocles, obra que, junto a formas en -el- presenta una 3. ${ }^{\mathrm{a}} \mathrm{pl} . \dot{\varepsilon} \omega \dot{\omega} \kappa \varepsilon \sigma \alpha \nu(20.6) .{ }^{129}$

\subsection{Imperativo: $3 \cdot{ }^{a}$ persona del plural}

Las terminaciones que presenta el ático son $-v \tau \omega \nu$ y $-\sigma \theta \omega \nu$, pero $-\tau \omega \sigma \alpha \nu$ y $-\sigma \theta \omega \sigma \alpha \nu$, creadas a partir de la desinencia jónica secundaria $-\sigma \alpha v,{ }^{130}$ aparecen junto a las primeras ya en la prosa clásica, por ejemplo en Tucídides, y hay también ejemplos en la tragedia, en Jenofonte y en Eneas Táctico. ${ }^{131}$ Pero a partir de 352/1 - $\tau \omega \sigma \alpha v$ está atestiguada en las inscripciones, ${ }^{132} \mathrm{y}$ en griego helenístico $-\tau \omega \sigma \alpha \nu$ y $-\sigma \theta \omega \sigma \alpha \nu$ se hacen mayoritarias, siendo ya hegemónicas en los papiros, así como en el $N T .^{133}$

En los autores aticistas podemos encontrar ambas terminaciones. ${ }^{134}$ No parece que la presencia de $-\tau \omega \sigma \alpha \nu$ se pueda considerar aticismo, pese a aparecer en autores clásicos como Tucídides, etc., sino que quizá tiene más sentido pensar que su presencia se deba a influencia de la koiné, ya que $-v \tau \omega \nu$ es exclusivamente ática, es decir, el aticismo puro al cual, lógicamente, habrían recurrido los aticistas. ${ }^{135}$ No obstante, quizá la presencia de $-\tau \omega \sigma \alpha \nu$ en autores clásicos, aun siendo minoritaria, haya facilitado su uso

${ }^{125}$ Cf. en general Chantraine, Morph. $\$ 232$; la conjugación ática, por lo tanto, responde a un modelo

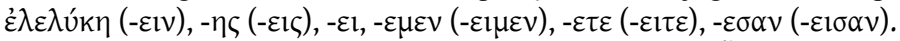

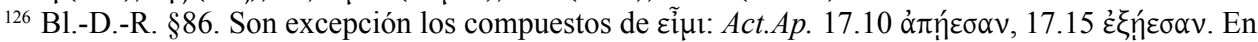

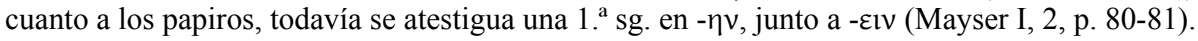

${ }^{127}$ Schmid, Atticismus IV, 27.

${ }^{128}$ Es el único ejemplo de 3. ${ }^{a}$ pl. que hemos encontrado (3.10.2). Respecto del sg., podemos citar a

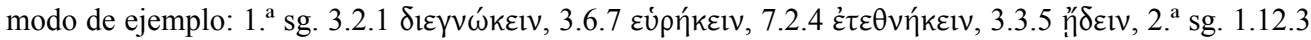

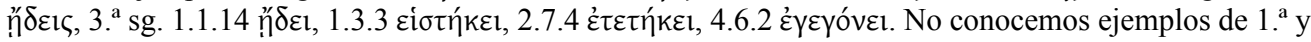
2. ${ }^{\mathrm{a}} \mathrm{pl}$.

${ }^{129}$ Vicente Sánchez (2006: 135-136); solo faltan los ejemplos para la 2. ${ }^{a}$ pl.

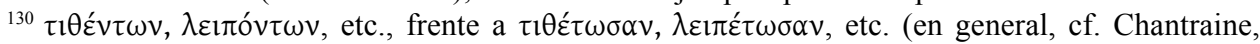
Morph. §318).

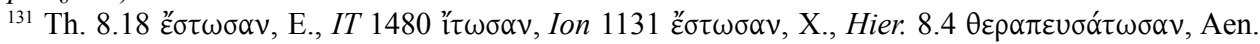

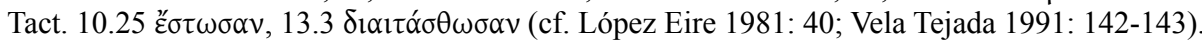

132 Threatte (1996: 463-467); casi todos los ejemplos son de presente o aoristo activo, aunque están atestiguados algunos ejemplos de aoristo pasivo.

${ }_{133}$ Mayser I, 2, p. 89; Bl.-D.-R. $\S 84_{1}$.

${ }^{134}-v \tau \omega \nu$ es la forma usada por Aristides y Filóstrato, mientras que Eliano usa - $\tau \omega \sigma \alpha v$ y Luciano ambas: cf. Schmid, Atticismus I, 229-230; II, 26-27; III, 32; IV, 27 y 589 (resumen). El único ejemplo de las Cartas de Temístocles es - $\tau \omega \sigma \alpha v$ (20.11), cf. Vicente Sánchez (2006: 136).

${ }^{135}$ Por eso Schmid, Atticismus IV, 597, cree que $-\tau \omega \sigma \alpha v$ es un vulgarismo en los autores aticistas. 
a autores aticistas (por ejemplo, Luciano, que usa ambas posibilidades), conscientes de que, al hacerlo, no estaban infringiendo una regla de la lengua modelo.

En todo caso, y dado que Caritón presenta solo formas en $-\tau \omega \sigma \alpha \nu$ y en número no exiguo, ${ }^{136}$ parece lógico que se trate de un rasgo de koiné.

\subsection{Aoristo}

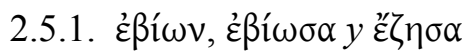

El aoristo sigmático o en - $\sigma \alpha$ fue convirtiéndose con el tiempo en la forma «normal» del aoristo griego, en el sentido de que fue imponiéndose al aoristo radical. ${ }^{137}$ Así sucede en el verbo ßıó $\omega$, cuyo aoristo radical es frecuente en la prosa ática, pero va dando paso a $\varepsilon \varepsilon_{i} \omega \sigma \alpha$, atestiguado ya en Heródoto, Platón o Jenofonte. ${ }^{138}$ En griego

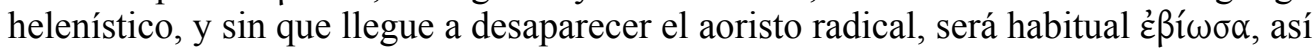
como ع̌ そ̌ $\sigma \alpha$, dándose ambos aoristos sigmáticos en el $N T .{ }^{139}$ Los papiros de época romana presentan $\varepsilon \dot{\beta} \hat{i}_{\omega} \omega \alpha$ de manera habitual (también $\left.\varepsilon^{\prime} \zeta \eta \sigma \alpha\right)$, pero el aoristo radical también está atestiguado. ${ }^{140}$ En los escritores aticistas el panorama es diverso, apare-

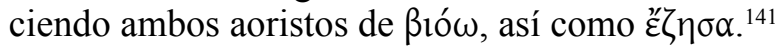

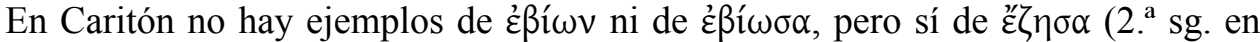
4.4.4 y 5.2.5, 3. ${ }^{\mathrm{a}} \mathrm{sg}$. en 7.5.4). Sin embargo, en 5.10.1 aparece $\alpha$ $v \varepsilon \beta i ́ \omega v$. Tenemos aquí, por lo tanto, un rasgo que apunta al ático antiguo; sin embargo, la no presencia de

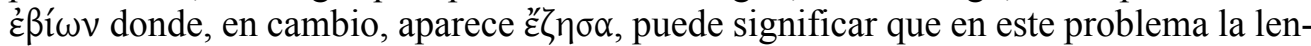
gua de Caritón no es aticista de manera consistente, sino que se limita a elegir la forma

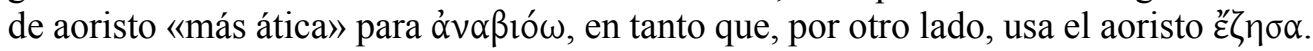

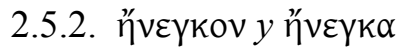

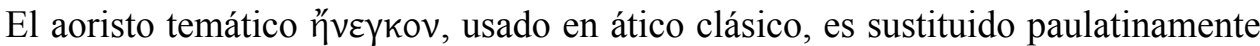

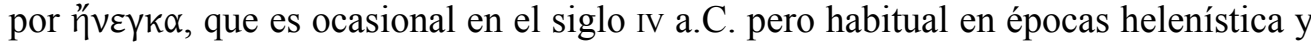
romana; en los papiros de estas épocas será muy mayoritario, al igual que en el $N T .^{142}$

En Caritón encontramos, junto a formas de infinitivo y participio, la $3 .^{\mathrm{a}}$ pers. sg. y pl. de indicativo, más un caso de $1 .^{\mathrm{a}} \mathrm{sg}$. y otro de $3 .^{\mathrm{a}} \mathrm{sg}$. de subjuntivo. La 3. ${ }^{\mathrm{a}}$ pers. sg., ฑँ $v \varepsilon \gamma \kappa \varepsilon$, y las formas de subjuntivo no sirven para discriminar por ser comunes a ambos aoristos. ${ }^{143}$ Por lo tanto, solo la $3 .^{\text {a }}$ pers. pl. ind. es pertinente; los

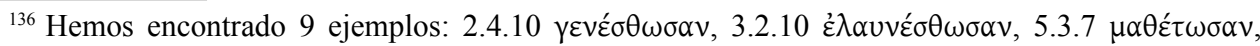

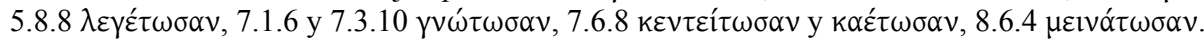

${ }^{137}$ En general, cf. Chantraine, Morph. $\$ 205$.

${ }^{138}$ Hdt. 1.163; Pl., Ph. 113d; X., Oec. 4.18 (cf. Gautier 1911: 64).

139 Bl.-D.-R. $§ 75_{2}$.

${ }^{140}$ Gignac (1981: 293).

${ }^{141}$ Schmid, Atticismus I, 231; III, 39-40; IV 25, 600.

${ }_{142}$ Mayser I, 2, pp. 136-137; Gignac (1981: 338-340); B1.-D.-R. §81 2 ; también Chantraine, Morph. §184.

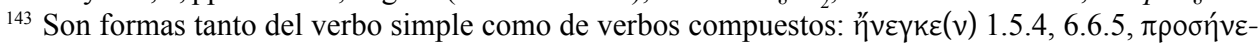

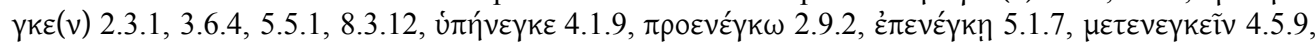

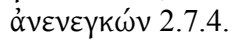




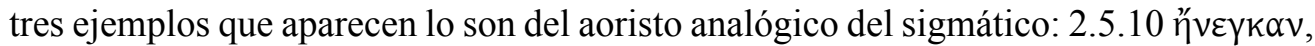

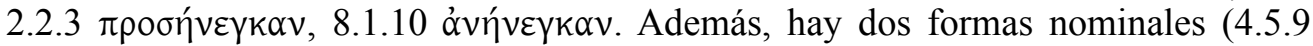
$\mu \varepsilon \tau \varepsilon v \varepsilon \gamma \kappa \varepsilon \tilde{\imath} v, 2.7 .4 \dot{\alpha} v \varepsilon v \varepsilon \gamma \kappa \omega \dot{v} v$ ), que corresponden al aoristo temático. Esta es una distribución habitual en autores aticistas,${ }^{144}$ sin que ello revele un gran rigorismo

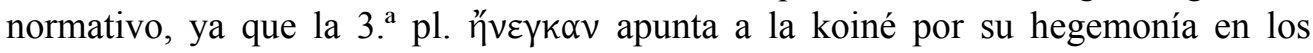
papiros. ${ }^{145}$

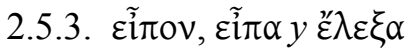

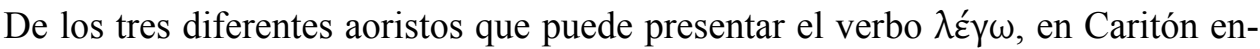

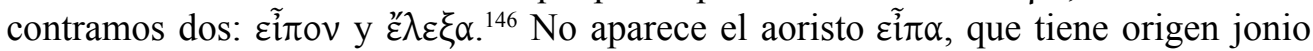
y puede sustituir al aoristo radical temático ya desde época clásica, ${ }^{147}$ si bien عĩ continúa usándose, sobre todo en las formas nominales; en Tucídides y Jenofonte es exclusivo en estas formas. En cuanto a los aticistas, usan sobre todo el aoristo temático, incluso de forma exclusiva, como sucede con Eliano. ${ }^{148}$

El aoristo $\varepsilon^{\prime} \lambda \varepsilon \xi \alpha$ está atestiguado en prosistas clásicos con el significado de «decir», ${ }^{149}$ pero con este significado no aparece ya ni en los papiros ptolemaicos ni en el $N T .{ }^{150}$ En Caritón está cuatro veces (7.3.1, 7.3.7, 8.1.6, 8.2.9), tres de ellas como introducción del discurso directo de un personaje: el soberano de los rebeldes egipcios en alocución a los miembros de su Consejo (7.3.1) y Quéreas dirigiéndose a sus soldados (7.3.7 y 8.2.9). Es más, en estos tres casos aparece bajo la fórmula $\varepsilon^{\prime} \lambda \varepsilon \xi \varepsilon v \tilde{\omega} \delta \varepsilon$, seguida del discurso que comienza siempre por el vocativo ő้ $\delta \rho \varepsilon \varepsilon$. Parece claro que se trata de una expresión formularia tomada de contextos similares de autores clásicos, que en este caso son muy probablemente Tucídides ${ }^{151}$ y, quizá más aún, Jenofonte, quien usa con frecuencia y en distintas obras la expresión $\varepsilon^{\prime} \lambda \varepsilon \xi \varepsilon v \tilde{\omega} \delta \varepsilon$ o $\left.\tilde{\omega} \delta^{\prime} \check{\varepsilon}^{\prime} \lambda \varepsilon \xi \varepsilon v\right) .{ }^{152}$

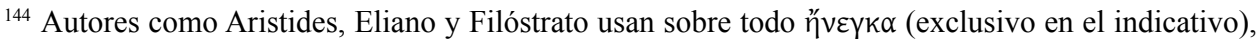
pero solo el primero emplea también las correspondientes formas nominales; Luciano, en cambio, suele

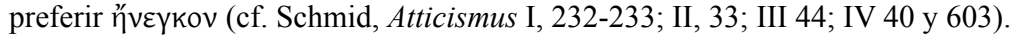

${ }^{145}$ En las Cartas de Temístocles aparecen solo las formas antiguas, que son dos, ambas nominales y

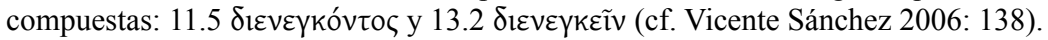

${ }^{146}$ Este último aparece en cuatro ocasiones, siempre con el significado de «decir», y nunca con el de «reunir», el único que tenía originariamente en la prosa clásica.

${ }^{147}$ Por ejemplo en Aristófanes, donde aparece junto a عĩ̄ov, incluso en la misma obra: Ach. 579 y 152; se trata de un ejemplo entre muchos de dobletes en los que, junto al arcaísmo, se da la forma moderna, nivelada con el jónico o bien simplificada y regularizada (López Eire 1986: 249-250).

${ }^{148}$ Schmid, Atticismus I, 232; II, 31; IV, 38 y 603.

${ }^{149}$ Pero es minoritario en comparación con عĩtov. De la persona verbal más común con mucho, $\varepsilon \varepsilon^{\lambda} \varepsilon \xi \varepsilon(v)$, gracias al $T L G$ se cuentan 8 ejemplos en Tucídides, frente a 26 de $\varepsilon \tilde{i} \pi \varepsilon(v)$, y 104 en Jenofonte, frente a 525 del aoristo temático.

${ }^{150}$ Mayser I, 2, p. 185; B1.-D.-R. $\$ 101_{48}$

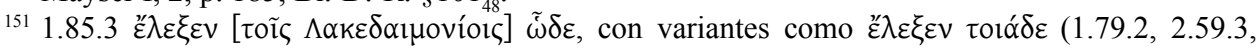

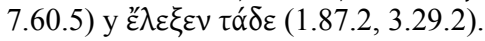

${ }^{152} H G$ 2.3.24, An. 3.2.2, 3.2.4, 5.1.2, Cyr. 2.2.17, 2.4.21, 3.3.7, etc. En todos los casos citados el dis-

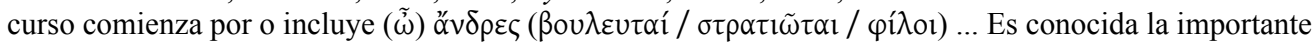
influencia de Jenofonte en Caritón, especialmente de la Ciropedia. 
Por lo tanto, todo parece indicar que el uso de $\varepsilon$ ’’ $\varepsilon \xi \xi v$ por parte de Caritón se debe a una elección concreta de tipo estilístico. Unido esto a la ausencia de $\varepsilon \tilde{i} \pi \alpha$, se puede hablar en conjunto de una elección de tipo aticista o, al menos, clasicista.

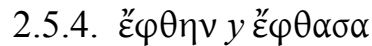

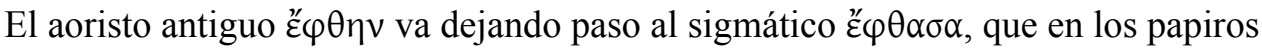

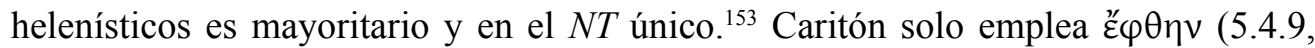
5.5.6 y 6.1.11), lo que apunta al modelo aticista. ${ }^{154}$

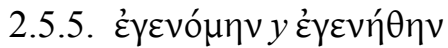

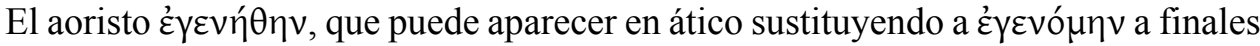
del siglo Iv a.C., después se propaga en la koiné. ${ }^{155}$ En autores tardíos y aticistas apa-

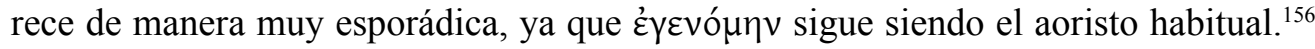
No es significativo, por tanto, que en Caritón aparezcan solo las formas de દ̇үદvó $\mu \eta v$ (16 veces en total).

En resumen, se puede decir con respecto al aoristo que Caritón presenta un panorama diverso. Quizá son mayoría las formas áticas genuinas, como عĩ̄ov (y no

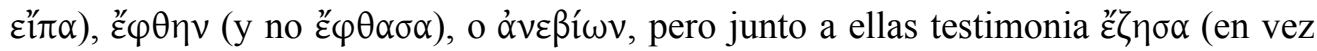
de $\varepsilon \beta i ́ \omega v)$ y también de koiné.

\subsection{Futuro $^{157}$}

\subsubsection{Futuro ático}

A la hora de optar entre el llamado «futuro ático», es decir, el futuro contracto

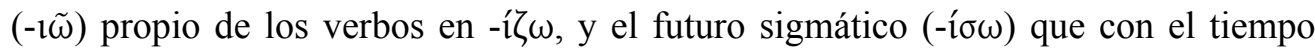
lo va sustituyendo, ${ }^{158}$ y que en la koiné neotestamentaria ya es hegemónico, ${ }^{159}$ Cari-

${ }^{153}$ Mayser I, 2, pp. 142 y 200; B1.-D.-R. $§ 101_{82}$.

${ }^{154} \mathrm{Si}$ lo comparamos con los demás novelistas vemos que en estos hay, por el contrario, una mezcla o incluso el predominio del aoristo sigmático. Heliodoro presenta el uso más ático, con 8 ejemplos del aoristo $\varepsilon ้ \varphi \theta \eta v$ y 4 del infinitivo $\varphi \theta \tilde{\eta} v \alpha$, pero también dos veces $\tilde{\varepsilon} \varphi \theta \eta \sigma \alpha \nu$ y una el participio sigmático

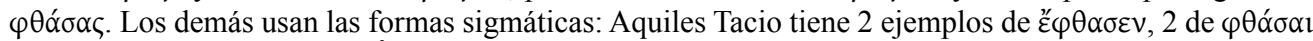
y 7 de $\varphi \theta \alpha ́ \sigma \alpha \varsigma$, Jenofonte de Éfeso y Longo solo un ejemplo, ambos del participio $\varphi \theta \alpha ́ \sigma \alpha \varsigma$.

${ }^{155}$ Chantraine, Morph. $\$ 192$.

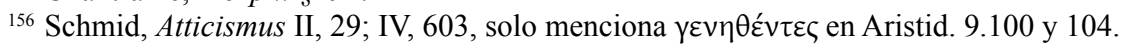

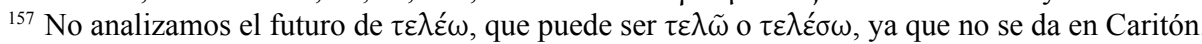

${ }^{158}$ Ya en época clásica hay algunas vacilaciones: en general, cf. Chantraine, Morph. \$297.

159 B1.-D.-R. §74.1; «-l $\tilde{\omega}$ fast nur in Zitaten aus LXX» (B1.-D.-R. §74 4 ). 
tón se inclina por el primero en las dos ocasiones pertinentes: $1.2 .5 \dot{\varepsilon} \varphi \circ \pi \lambda \mathrm{i} \tilde{\omega}, 2.11 .6$ $\dot{\varepsilon} \xi o \rho \kappa \imath \tilde{\omega} .{ }^{160}$ Por lo tanto, coincide con la práctica normal en los autores aticistas. ${ }^{161}$

\subsection{2. $\mu \alpha \chi о \tilde{v} \mu \alpha 1$ y $\mu \alpha \chi \eta ́ \sigma o \mu \alpha 1$}

Frente al futuro contracto $\mu \alpha \chi o \tilde{v} \mu \alpha$, que es la forma antigua, y habitual en los prosistas clásicos, la koiné favorece el tipo sigmático $\mu \alpha \chi \eta ́ \sigma o \mu \alpha l$, presente ya desde Homero. ${ }^{162}$ Después, $\mu \alpha \chi 0 \tilde{\mu} \mu \alpha$ será lo habitual en los autores aticistas. ${ }^{163}$

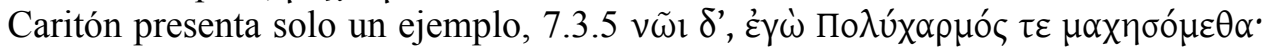

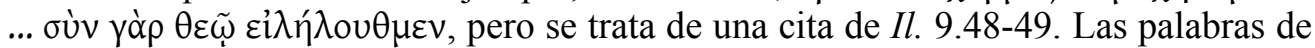
Diomedes refiriéndose a Esténelo ( $\varepsilon_{\gamma} \dot{\omega} \Sigma \theta \varepsilon \dot{v} \varepsilon \lambda$ ó $\left.\tau \varepsilon \mu \alpha \chi \eta \sigma o ́ \mu \varepsilon \theta \alpha\right)$ y a sí mismo son adaptadas por Caritón para que Quéreas se refiera a Policarmo. Por tanto, está claro que la forma verbal empleada es la homérica, lo que invalida el ejemplo para discernir cuál era el futuro preferido por Caritón.

\subsection{El perfecto: $\delta \varepsilon ́ \delta o เ \kappa \alpha y \delta \varepsilon ́ \delta ı \alpha$}

La flexión habitual en ático del perfecto de $\delta \varepsilon i ́ \delta \omega^{164}$ es $\delta \varepsilon ́ \delta o \imath k \alpha,-\alpha \varsigma,-\varepsilon, \delta \varepsilon \delta \delta \mu \varepsilon v$,

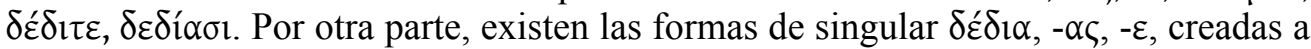

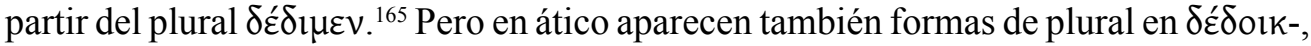

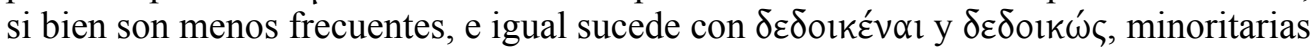

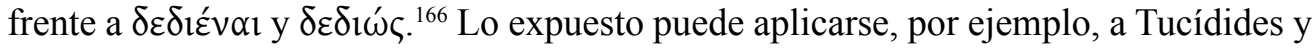
Jenofonte, los dos prosistas que son los principales modelos de Caritón. ${ }^{167}$

Añadamos que los papiros de época romana atestiguan ambos participios, ${ }^{168} \mathrm{y}$ también en los autores aticistas parecen darse ambas posibilidades, también en el perf.

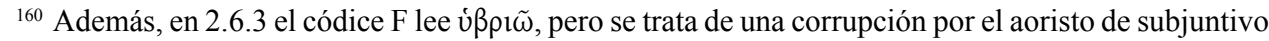
úßpí $\sigma \omega$ (enmienda de Cobet 1859: 260), ya que el pasaje requiere un subjuntivo deliberativo.

${ }^{161}$ Aristides y Eliano presentan algunas formas sigmáticas; Luciano y Filóstrato solo las contractas:

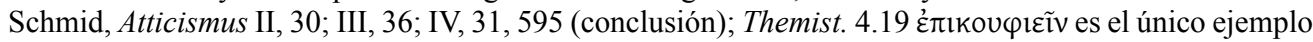

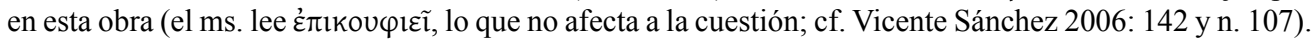

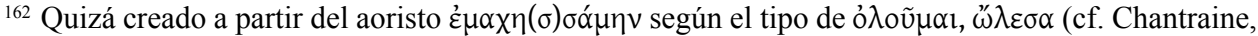
Morph. §296).

${ }^{163}$ Incluso un autor no aticista como Plutarco da preeminencia a $\mu \alpha \chi 0 \tilde{v} \mu \alpha l$, si bien usa $\mu \alpha \chi \eta ́ \sigma o \mu \alpha l$ (cf. Weissenberger 1994: 36). Las Cartas de Temístocles presentan solo $\mu \alpha \chi 0 \tilde{u} \mu \alpha \mathrm{l}$, con 4 ejemplos (cf. Vicente Sánchez 2006: 141).

${ }^{164}$ Que es, a su vez, un antiguo perfecto, $* \delta \varepsilon \delta$ woy- $\alpha$ (Chantraine, Morph. §218).

${ }^{165}$ Chantraine, Morph. §218, que da una explicación poco clara, y K.-Bl. II, 401-402. La flexión habitual del ático se halla, por ejemplo, en Tucídides (véase n. 167). $\delta \varepsilon ́ \delta 1 \alpha$ está ya en A., Pr. 182, 902 (lír.).

${ }^{166}$ Véase en general K.-Bl. II, 402, con ejemplos.

${ }^{167}$ Una búsqueda mediante el $T L G$ da como resultado que Tucídides solo usa $\delta \varepsilon \delta$ lév@l (4 veces) y

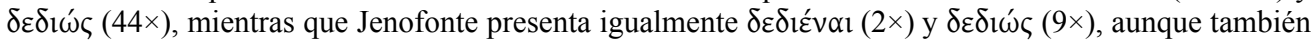

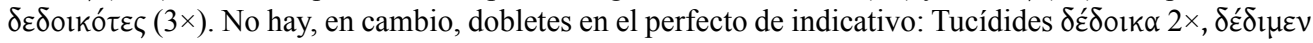
$3 \times, \delta \varepsilon \delta \delta \tau \varepsilon 1 \times$, Jenofonte $\delta \varepsilon ́ \delta \circ \imath \kappa \alpha 9 \times,-\alpha \varsigma 1 \times, \delta \varepsilon ́ \delta \imath \tau \varepsilon 1 \times, \delta \varepsilon \delta i ́ \alpha \sigma \iota 1 \times$. Es decir, la conjugación «regular» del ático clásico.

${ }^{168}$ Gignac (1981: 299); no hay ejemplos de perfecto o pluscuamperfecto de indicativo. Mayser no recoge ejemplos de $\delta \varepsilon i ́ \delta \omega$ para los papiros de época helenística. 
ind. ${ }^{169}$ La lengua del $N T$ no sirve de ayuda, ya que no usa $\delta \varepsilon i ́ \delta \omega .{ }^{170}$ En resumen, un panorama complejo, motivo por el cual, aunque se ha analizado la aparición de ambos tipos como rasgo distintivo de aticismo o koiné, ${ }^{171}$ lo cierto es que tal análisis presenta dificultades. No obstante, de forma general, especialmente si nos atenemos a la prosa, de donde fundamentalmente bebe Caritón, se puede decir en resumen que lo ático, por más frecuente, es el perf. ind. ya indicado al comienzo de este apartado, acompañado de las formas nominales $\delta \varepsilon \delta 1 \varepsilon ́ v \alpha ı$ y $\delta \varepsilon \delta ı \omega ́ c$.

En Caritón hay ejemplos de ambas formas. Por un lado, las formas en Solk- (10 ejemplos), tanto de indicativo como infinitivo y participio, ${ }^{172}$ pero junto a ellas las formas en grado cero, si bien en menor número (4 ejemplos) y solo nominales: $\delta \varepsilon \delta 1 \varepsilon ́ v \alpha 1$

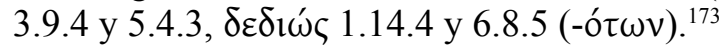

Por tanto, se puede afirmar que el novelista no se aparta de la norma clásica cuan-

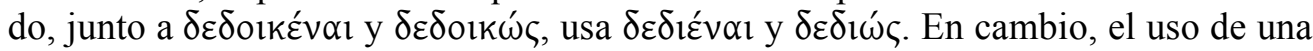
forma de plural del perf. ind. como $\delta \varepsilon \delta$ oík $\alpha \mu \varepsilon v$ (7.3.3) resulta hasta cierto punto chocante, ya que, aunque las formas plurales en $\delta \varepsilon ́ \delta o l k-$ están atestiguadas en autores clásicos, ${ }^{174}$ son poco habituales, además de ajenas a la conjugación ática «regular»: por ejemplo, no aparecen en prosistas como Tucídides y Jenofonte. ${ }^{175}$ Cabe pensar en una influencia literaria, si se tiene en cuenta que $\delta \varepsilon \delta o$ ó $\alpha \mu \varepsilon v$ está en Menandro (fr. 534 K.), un autor muy presente en Caritón. Otra posibilidad, compatible con la

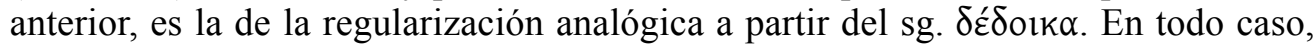
lo que interesa destacar para nuestro estudio es que Caritón no sigue una norma aticista a rajatabla, ya que mezcla ambos tipos de formas y, por otra parte, no respeta la conjugación del perfecto de indicativo propia de la prosa ática, ya que extiende $\delta \varepsilon ́ \delta$ oık- al plural.

${ }^{169}$ Schmid, Atticismus IV, 33-34, 601-602): el sg. $\delta \varepsilon ́ \delta 1 \alpha,-\alpha \varsigma,-\varepsilon$ está en Luciano y, sobre todo, en Filóstrato.

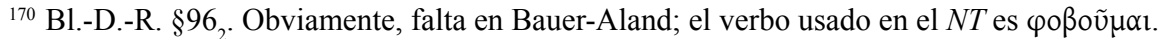

${ }^{171}$ Lo serían las formas en $\delta \varepsilon ́ \delta o l k-$ y en $\delta \varepsilon ́ \delta$-, respectivamente. Así, Vicente Sánchez (2006: 142) con respecto a las Cartas de Temístocles, sin llegar a una conclusión fehaciente. Como se podrá apreciar, tal distribución es discutible.

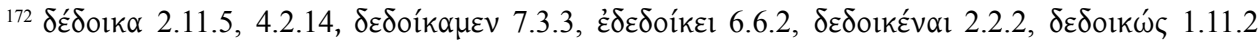
(-vĩ $\alpha), 3.9 .6,4.2 .11,4.7 .1,5.2 .1$.

${ }^{173}$ Añadamos (4.2.14) el imperativo en grado cero $\delta \varepsilon ́ \delta 1 \theta \imath$ (cf. Chantraine, Morph. §315), que está en ático (Ar., V. 373, Eq. 230) y aparece en la prosa tardía (cf. K.-Bl. II, 402).

174 Ar., Ec. $141 \delta \varepsilon \delta o$ ík $\alpha \tau \varepsilon$, D., Ep. $3.33 \delta \varepsilon \delta$ oík $\alpha \sigma$ (cf. K.-B1. II, 402).

${ }^{175}$ Quienes sí tienen las formas de plural en $\delta \varepsilon ́ \delta$ l- (véase n. 167). Por otro lado, un autor aticista como Filóstrato (véase n. 169) no lo usa, ya que limita $\delta \varepsilon ́ \delta o เ k-a l$ singular, y $\delta \varepsilon ́ \delta$ - lo usa para el plural, pero lo extiende al singular; además, usa ambas posibilidades para las formas nominales, en este caso igual que Caritón. Si nos fijamos en los demás novelistas, encontramos el panorama esperable, con mezcla de

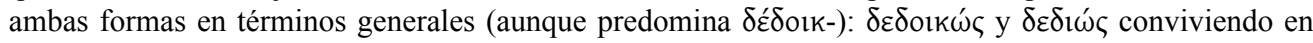
Jenofonte, Longo y Heliodoro, sin ejemplos en Aquiles Tacio; del infinitivo solo hay un ejemplo en Hel.

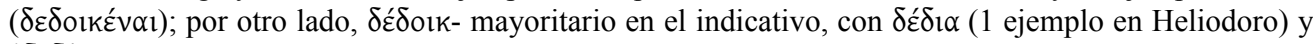
$\varepsilon \dot{\varepsilon} \delta \delta$ ícl $(2 \times$ en A.T. y $1 \times$ en Hel.), pero no es posible conocer el plural del perfecto, porque ningún autor ofrece ejemplos. 


\subsection{El llamado optativo eolio}

En época clásica el ático utiliza por lo general el llamado «optativo eolio», es decir, el aoristo sigmático de voz activa que, junto a $-\sigma \alpha \iota \mu l,-\sigma \alpha \iota \mu \varepsilon v$ y $-\sigma \alpha \iota \tau \varepsilon$, incluye la $2 .^{\mathrm{a}} \mathrm{y}$

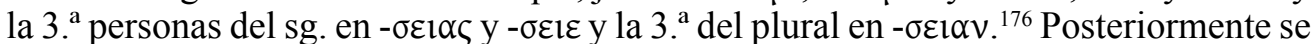
irán imponiendo las formas regulares en $-\sigma \alpha 1 \varsigma,-\sigma \alpha l$ y $-\sigma \alpha l \varepsilon v$, que serán hegemónicas en los papiros de época helenística. ${ }^{177} \mathrm{Sin}$ embargo, el movimiento aticista favorece de nuevo el optativo eolio. ${ }^{178}$

El panorama en Caritón es claro, ya que para la 3. ${ }^{a} \mathrm{sg}$. y 3..$^{\mathrm{a}}$ pl. (la 2. ${ }^{\mathrm{a}} \mathrm{sg}$. no existe) solo usa las formas eolias, con 7 ejemplos en total. ${ }^{179}$ Por lo tanto, en este caso estamos ante un rasgo aticista. ${ }^{180}$

Sin embargo, a este dato hay que añadir otro de carácter sintáctico que lo matiza. Como es sabido, el optativo ve reducido su uso en la koiné, y en el $N T$ es ya muy raro. ${ }^{181}$ Por lo tanto, su uso se convierte en un rasgo de aticismo, y como tal es acogido por los autores aticistas. Sin embargo, los datos parecen indicar que Caritón usa poco el optativo, o al menos así se deduce del análisis realizado por Papanikolaou, quien, al comparar la frecuencia de aparición de este modo verbal en nuestro autor con la de los demás novelistas, concluye que es quien menos usa el optativo, con considerable diferencia. ${ }^{182}$

\subsection{Tematización de verbos atemáticos}

En ático clásico se da ya el fenómeno de la tematización de verbos atemáticos. Un autor como Jenofonte presenta no pocos ejemplos del fenómeno, en lo que se ha visto al historiador como un precursor de la koiné. ${ }^{183} \mathrm{Y}$ si la koiné acoge las formas tematizadas, los autores aticistas las evitan, para regresar a las formas atemáticas como propias y genuinas del ático. ${ }^{184}$

${ }^{176}$ Ambas series coexisten ya en Homero; cf. Chantraine, Morph. $\$ 313$.

${ }^{177}$ Mayser I, 2, 87-88. En el $N T$ la 3. ${ }^{\text {a }}$ sg. es $-\sigma \alpha$ l, mientras que de la 3. ${ }^{a}$ pl. hay un ejemplo de cada serie (Bl.-D.-R. $\S 85_{2}$ ). Téngase en cuenta que el optativo es un modo cuyo uso va decayendo paulatinamente en griego, siendo ya casi extraño al $N T$ (B1.-D.-R. $§ 65,2)$.

${ }^{178}$ Schmid, Atticismus IV, 588.

${ }^{179} 3 .^{\mathrm{a}}$ sg.: $1.1 .12,3.8 .8,4.7 .2 \dagger, 4.7 .4,7.6 .9 ; 3 .^{\mathrm{a}}$ pl.: 7.4.2, 8.3.2. De las formas regulares no hemos encontrado ningún ejemplo.

${ }^{180}$ Coincide con el autor de las Cartas de Temistocles, obra que presenta solo el optativo eolio, con 7 casos (cf. Vicente Sánchez 2006: 143).

${ }^{181}$ Véase n. 177.

182 Papanikolaou (1973: 90). En la muestra analizada, consistente en 70 páginas de Teubner (se refiere a los Erotici Scriptores Graeci de Hercher, 1858-1859), Caritón tiene 20 ejemplos de optativo, Aquiles Tacio 36, Longo 37, Jenofonte 71 y Heliodoro 94. En el total de la obra de Caritón (ocupa 155 páginas en la edición de Hercher) hay un total de 51 optativos.

${ }^{183}$ La proporción de estos verbos es superior en Jenofonte a la de sus contemporáneos, lo que sería un ejemplo de anticipación del uso helenístico (cf. Gautier 1911: 82, quien cita este rasgo junto a otros).

${ }^{184}$ No nos detendremos en pormenores, como por ejemplo si el fenómeno se da más en la voz activa o en la media y la pasiva, lo que a la vista de los ejemplos de Caritón carece de importancia; para los autores aticistas, cf. Schmid, Atticismus I, 52, 83, 229; II, 25; III, 37; IV, 31-32, 595-596 (conclusión), 
En Caritón destacaremos dos hechos. El primero, que es posible encontrar tanto formas atemáticas como formas tematizadas, en una proporción bastante pareja. ${ }^{185} \mathrm{El}$ segundo, que la coexistencia de ambos tipos se da incluso en el mismo verbo (o un

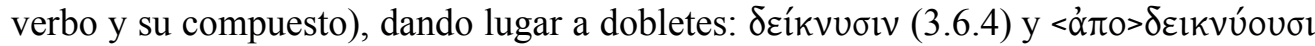

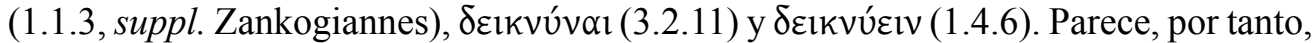
que el novelista no se preocupa mucho por mantener o rescatar las formas atemáticas. ${ }^{186} \mathrm{El}$ uso de las formas tematizadas, en cambio, apunta a un rasgo de koiné. El hecho de que este rasgo quede atenuado por la existencia también de formas atemáticas puede significar que nos hallemos ante una tendencia a expresar formas propias de la lengua clásica, pero no ante una norma.

\section{CONCLUSIONES}

No parece que la lengua de Caritón en su vertiente morfológica siga de manera estricta las normas del ático, si nos atenemos a los datos examinados. Existe, como es lógico, una tendencia a usar formas áticas, pero tales formas aparecen a menudo junto a sus equivalentes de la koiné, y otras veces son estas las únicas usadas.

Veamos ahora de manera sinóptica las formas que pueden ser consideradas aticismos, recordando los datos esenciales de cada caso. ${ }^{187}$ Junto a veẃs $(5 \times)$ aparece la forma correspondiente de la koiné, vaós $(1 \times)$. Junto a la forma contracta $\chi \rho v \sigma \tilde{\eta} v(2 \times)$ aparecen $\chi \rho u ́ \sigma \varepsilon o v$ y $\chi \rho u ́ \sigma \varepsilon \alpha(2 \times$ y $1 \times)$. La forma $\theta \alpha ́ \tau \varepsilon \rho o v$ se da 4 veces, pero hay un

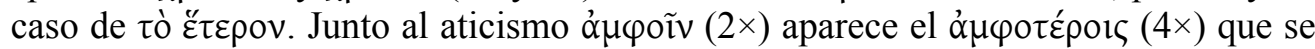

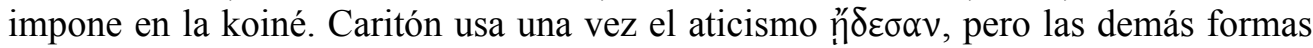
de pluscuamperfecto son en -El-. Con respecto a las formas de aoristo encontramos un panorama variopinto, con formas verbales propias del ático frente a términos tardíos, que no repetimos aquí: puede verse la conclusión al final del apartado 2.5.5. Un uso genuinamente ático es el del futuro contracto en $-i \tilde{\omega}$. Por último, otro rasgo también ático es el del llamado optativo eolio, lo que queda matizado por el hecho de que Caritón usa muy poco el optativo, siguiendo la tendencia histórica de la lengua griega a eliminar este modo verbal. Aparecen también los presentes tematizados, como $\delta \varepsilon ı v v u ́ \omega$, pero sin que hayan desaparecido las formas atemáticas; el reparto es parejo,

605. Léxicos aticistas, como los de Meris y Frínico, explicitan que las formas atemáticas son las propias del ático (Schmid, Atticismus I, 52, 83).

${ }^{185}$ A partir de un análisis no completamente exhaustivo podemos mencionar formas atemáticas como

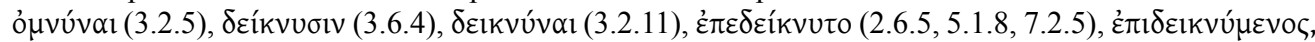

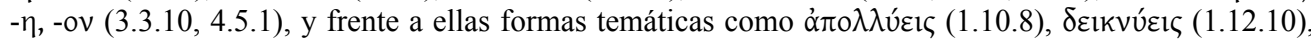

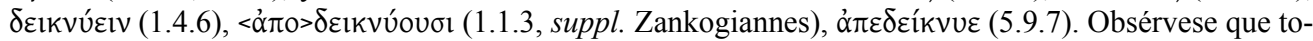
das las formas de la voz media son atemáticas, mientras que las activas son tanto atemáticas como temáticas.

${ }^{186}$ A diferencia de lo que hace el autor de las Cartas de Temístocles, si bien en esta obra también hay ejemplos de ambos tipos (cf. Vicente Sánchez 2006: 145).

${ }^{187}$ No haremos alusión de nuevo a las formas propias del ático que se mantienen en la koiné, ya que no son significativas para caracterizar la lengua del autor. 
y se dan incluso los dobletes, es decir, la aparición de una forma atemática junto a la correspondiente temática.

Por otro lado, la lengua de Caritón presenta un buen número de características morfológicas propias de la koiné, sin que aparezca en estos casos la forma ática equi-

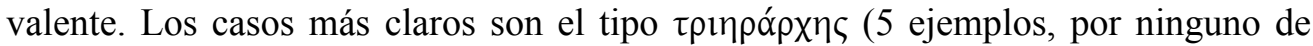

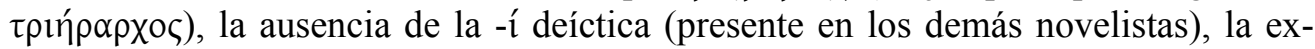

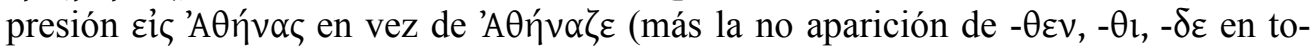
pónimos), las segundas personas oĩ $\delta \alpha \varsigma$ (por oĩ $\sigma \theta \alpha$ ) y $\tilde{\eta} \varsigma$ (por $\tilde{\eta} \sigma \theta \alpha$ ), la desinencia de imperativo $-\tau \omega \sigma \alpha v$ supliendo a la clásica $-v \tau \omega v$, la $3 .^{a}$ pers. pl. de aoristo

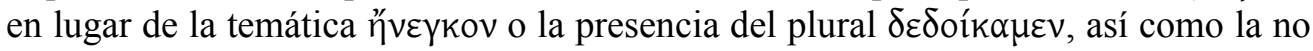
existencia del dual en la conjugación. Son casos en los que Caritón no ha aplicado en absoluto el rigor aticista.

La primera conclusión que puede extraerse a partir de los datos analizados es que la lengua de Caritón presenta un buen número de características morfológicas propias de la koiné helenística, en algunos casos coexistiendo con las correspondientes características áticas, pero en otros casos con carácter exclusivo.

Pero, dicho esto, lo cierto es que los aticismos existen, sin que se trate de formas testimoniales o anecdóticas. Una forma de explicar este fenómeno consiste en que, en la etapa previa a la hegemonía aticista, la tendencia a imitar la prosa clásica existía ya. En otras palabras, antes de que el aticismo actuara como una corriente normativa plenamente establecida, los autores tardíos mostraban ya una tendencia a imitar la lengua de los prosistas clásicos; no hay que olvidar que son lenguas literarias. ${ }^{188}$ No se trataría de una mera adopción ocasional de términos literarios, expresiones arcaizantes, citas, etc., tomados de los grandes autores del pasado, ${ }^{189}$ sino de la aceptación de características fonológicas, morfológicas y sintácticas, además de léxico. Pero todo ello se habría producido de manera aún no sistemática, lo que explicaría la coexistencia de formas áticas y de sus formas correspondientes de koiné, incluidos dobletes. ${ }^{190} \mathrm{Si}$ esto es así, estaríamos ante una segunda conclusión del presente trabajo, que intentaría precisar en lo posible la primera conclusión ya expresada supra.

Por lo tanto, este estudio de la morfología de Caritón vendría a coincidir en sus resultados con estudios anteriores relativos a la fonología y el léxico. ${ }^{191}$ Por último, y en cuanto al tan debatido problema de la datación del novelista, una vez hecha la salvedad de que los textos literarios, por su propia naturaleza, no son prueba de un estadio cronológico concreto de lengua, este trabajo apunta en la dirección de que

${ }^{188}$ El término es classicisante, propuesto por Lasserre (1979), y aceptado o utilizado por diferentes especialistas.

${ }^{189}$ Por ejemplo, la palabra vaũ $\zeta$, que en la koiné ha sido sustituida por $\pi \lambda$ oĩov, pero que en Caritón (es algo normal en cualquier autor) aparece por razones puramente literarias. O bien citas de los grandes autores, como las abundantes citas homéricas (a veces adaptadas al propio texto) que usa el novelista.

${ }^{190}$ La existencia de dobletes ya fue observada por Hernández Lara (1994: 139-140), quien ofrece una lista de ejemplos, casi todos de carácter léxico.

${ }^{191}$ Sobre la fonología, cf. Sanz Morales (2014, conclusiones en pp. 299-301); sobre el léxico, cf. Ruiz Montero (1991) y Hernández Lara (1994, conclusión en p. 224). 
Caritón habría escrito posiblemente en la época previa a la eclosión aticista, no más tarde de las primeras décadas del siglo II d.C., pero tampoco en época muy anterior. ${ }^{192}$

\section{REFERENCIAS BIBLIOGRÁFICAS}

BAUER-Aland = W. Bauer, Griechisch-deutsches Wörterbuch zu den Schriften des Neuen Testaments und der frühchristlichen Literatur, Berlin-New York 1988. Unter Mitwirkung von V. Reichmann. Herausgegeben von K. Aland und B. Aland.

Blake, W.E. (1938), Charitonis Aphrodisiensis De Chaerea et Callirhoe Amatoriarum Narrationum Libri Octo, Oxonii.

BL.-D.-R. = F. Blass-A. Debrunner-F. Rehkopf, Grammatik des neutestamentlichen Griechisch, Göttingen $1984^{16}$.

Borgogno, A. (2005), Romanzi greci. Caritone d'Afrodisia. Senofonte Efesio. Longo Sofista, Torino.

Caballero López, J.A. (1997), La lengua y el estilo de la República de los atenienses del Pseudo-Jenofonte, Amsterdam.

Chantraine, $D E L G=$ P. Chantraine, Dictionnaire étymologique de la langue grecque, Paris 1968.

Chantraine, Morph. $=$ P. Chantraine, Morphologie historique du grec, Paris $1961^{2}$ (hay trad. esp., Reus 1974).

Coвet, C.G. (1859), «Annotationes Criticae ad Charitonem», Mnemosyne 8: 229-303.

CRESPO, E. (1979-80), «La alternancia $\Sigma \Sigma /$ TT y la prosa literaria ática del siglo v a.C.», $C F C$ 16: $109-126$.

D’Orville, J.P. $\left(1783^{2}\right)$, Charitonis Aphrodisiensis De Chaerea et Callirrhoe Amatoriarum Narrationum Libri VIII, Lipsiae (Amstelodami 1750'). Con comentario del ed. y Animadversiones y traducción latina de J.J. Reiske; 2. ${ }^{\mathrm{a}}$ ed. de C.A. Beck.

Foucault, J.-A. DE (1972), Recherches sur la langue et le style de Polybe, Paris.

Gautier, L. (1911), La langue de Xénophon, Genève.

Gignac, F.T. (1981), A Grammar of the Greek Papyri of the Roman and Byzantine Periods, vol. II. Morphology, Milano.

Goold, G.P. (1995), Chariton. Callirhoe, Cambridge Mass.-London.

Hercher, R. (1859), Erotici Scriptores Graeci, vol. II, Lipsiae.

Hernández Lara, C. (1994), Estudios sobre el aticismo de Caritón de Afrodisias, Amsterdam. Hirschig, G.A. (1856), Erotici Scriptores, Parisiis.

K.-BL. = R. Kühner-F. Blass, Ausführliche Grammatik der griechischen Sprache. Erster Teil: Elementar und Formenlehre, I-II, Hannover-Leipzig 1890-1892³ (= Darmstadt 1966).

${ }^{192}$ Es obvio que no pretendo resolver aquí el arduo problema de la datación del novelista, sino solo aportar un dato más al debate. En todo caso, me interesa señalar que mis conclusiones, al igual que las de los mencionados Ruiz Montero y Hernández Lara, contradicen la teoría de Papanikolaou (1973: 160 ss.), quien defendía que en Caritón no había huella alguna de aticismo, lo cual lo llevaba a datar al novelista en la segunda mitad del siglo I a.C. 
Lasserre, F. (1979), «Prose grecque classicisante», en Flashar, H. (ed.), Le classicisme à Rome aux I ${ }^{\text {ers }}$ siécles avant et après J.-C., Vandoeuvres-Genève: 135-163.

Lejeune, Phonétique = M. Lejeune, Phonétique historique du mycénien et du grec ancien, Paris 1972.

$L R o m G r=$ F. Conca-E. De Carli-G. Zanetto (I-II) y S. Beta-E. De Carli-G. Zanetto (III-IV), Lessico dei Romanzieri Greci, I-IV, Hildesheim-Zürich-New York 1989-2004 (vol. I = Milano 1983).

LóPEZ Eire, A. (1981), «Fundamentos sociolingüísticos del origen de la koiné», CFC 17: 21-53.

- (1984), «Tucídides y la koiné», en A. Bernabé-L.A. de Cuenca-E. Gangutia-J. López Facal, Athlon. Satura Grammatica in honorem Francisci R. Adrados, vol. I, Madrid: 245-261.

— (1986), «La lengua de la comedia aristofánica», Emerita 54: 237-274.

- (1991), Ático, koiné y aticismo. Estudios sobre Aristófanes y Libanio, Murcia.

López Martínez, M.P. (1998), Fragmentos papiráceos de novela griega, Alicante.

MAYSER = E. Mayser, Grammatik der griechischen Papyri aus der Ptolemäerzeit, I, Leipzig 1906, II.1 Berlin 1926, II.2 1934, II.3 1934.

Meckelnborg-SchäFer (2006) = C. Meckelnborg / K.H. Schäfer, Chariton. Kallirhoe, Darmstadt. Molinié, G. (1989²), Chariton. Chairéas et Callirhoé, ed. revisada por A. Billault, Paris (19791).

Papanikolaou, A.D. (1973), Charition-Studien. Untersuchungen zur Sprache und Chronologie der griechischen Romane, Göttingen.

Reardon, B.P. (2004), Chariton Aphrodisiensis. De Callirhoe Narrationes Amatoriae. Editionem curavit B.P.R. Monachii et Lipsiae.

Ruiz Montero, C. (1991), «Aspects of the Vocabulary of Chariton of Aphrodisias», CQ n.s. 41: 484-490.

Sanz Morales, M. (2014), «La lengua de Caritón de Afrodisias: características fonológicas», en Á. Martínez Fernández et al. (eds.), Ágalma. Ofrenda desde la Filología Clásica a Manuel García Teijeiro, Valladolid: 291-301.

Schmid, Atticismus = W. Schmid, Der Atticismus in seinen Hauptvertreten. Von Dyonisius von Halikarnass bis auf den zweiten Philostratus, I-V. Stuttgart 1887-1897 (reimpr. Hildesheim 1964).

SchwYZer = E. Schwyzer, Griechische Grammatik, I $\left(1953^{4}\right)$, II $\left(1966^{3}\right.$, vervollständigt und herausgegeben von A. Debrunner), III Register (1968³, von D.J. Georgacas), IV Stellenregister (1971, von F. Radt und S. Radt), München.

Threatte, L. (1996), The Grammar of Attic Inscriptions, II: Morphology, Berlin-New York.

$T L G=$ Thesaurus Linguae Graecae. A Digital Library of Greek Literature, Univ. of California Irvine: http://stephanus.tlg.uci.edu.

Vela Tejada, J. (1991), Estudio sobre la lengua de la Poliorcética de Eneas el Táctico, Zaragoza.

Vicente SÁnchez, A. (2006), Las Cartas de Temístocles. Lengua y técnica compositiva, Zaragoza.

Weinstein, M.E. (1972), Ed. de P. Oxy. 2948, en The Oxyrhynchus Papyri XLI, London: 12-14. Weissenberger, B. (1994), La lingua di Plutarco di Cheronea e gli scritti pseudoplutarchei, Napoli. A cura di G. Indelli; premessa di I. Gallo (ed. orig. alem., Straubing 1895). 